\title{
Live benthic foraminiferal faunas from the Bay of Biscay: faunal density, composition, and microhabitats
}

\author{
C. Fontanier*, F.J. Jorissen ${ }^{1}$, L. Licari ${ }^{2}$, A. Alexandre, P. Anschutz, P. Carbonel \\ Université Bordeaux, D.G.O. UMR 5805 CNRS, Avenue des Facultés, 33405 Talence Cedex, France
}

Received 28 February 2001; received in revised form 14 November 2001; accepted 16 November 2001

\begin{abstract}
In the meso-oligotrophic Bay of Biscay, a diminishing downward organic matter flux with depth is accompanied by an important decrease of the live foraminiferal density. Although bottom water oxygenation is not directly influenced by organic matter input, the oxygenation of interstitial waters and the primary redox fronts do change in response to variations of the organic matter flux. The occurrence of deep and intermediate infaunal taxa can be linked to fundamental redox fronts and putative associated bacterial consortia. Our data are in agreement with the TROXmodel, which explains the benthic foraminiferal microhabitat as a function of organic flux and benthic ecosystem oxygenation. Both the depth of the principle redox fronts and the microhabitat of deep infaunal species show important increases with depth. At the deepest oligotrophic stations, deep infaunal faunas become relatively poor. Therefore, the exported flux of organic matter appears to be the main parameter controlling the composition and the vertical distribution of benthic foraminiferal faunas below the sediment-water interface. The oxygenation of pore waters plays only a minor role. A species-level adaptation of the TROX-model is presented for the Bay of Biscay. (C) 2002 Elsevier Science Ltd. All rights reserved.
\end{abstract}

Keywords: Live benthic foraminifera; Exported organic matter flux; Redox conditions; Microhabitat

\section{Introduction}

Benthic foraminifera are an important component of the meiofaunal community of deep-sea detritus feeders. In deep-sea environments, they commonly represent more than $50 \%$ of the total biomass (Gooday et al., 1992). Thanks to their extraordinary potential of adaptation, benthic

\footnotetext{
*Corresponding author.

E-mail address: fontanier@hotmail.com (C. Fontanier).

${ }^{1}$ Present address: Angers University, Dept. Geology, UPRES EA 2644, 2 Boulevard Lavoisier, 49045 Angers, France.

${ }^{2}$ Present address: Alfred Wegener lust. for Polar and Marine Research, Columbusstrasse, D-27515 Bremerhaven, Germany.
}

foraminifera are able to survive and proliferate in a wide range of marine environments, including extreme ecosystems, such as oligotrophic abyssal plains (Tietjen, 1971; Coull et al., 1977) or hydrothermal vents (Sen Gupta and Aharon, 1994). Because of their potentially important role in deep ocean environments, they are at present studied intensively for a better understanding of their role in the benthic ecosystem and for a more precise definition of their contribution to the recycling of organic matter at the ocean floor.

There is a general consensus that the faunal composition of heterotrophic benthic foraminiferal faunas is strongly linked to the quantity and 
quality of the organic detritus reaching the ocean floor, and to the oxygenation at the sedimentwater interface and of the interstitial waters in the first $\mathrm{cm}$ of the sediment (e.g. Van der Zwaan, 1982; Altenbach and Sarnthein, 1989; Loubere et al., 1993; Fariduddin and Loubere, 1997; Jorissen et al., 1998). In this context, this paper describes the variability of live (Rose Bengal stained) benthic foraminiferal assemblages along a transect in the Bay of Biscay comprising five stations from 140 to $2000 \mathrm{~m}$ water depth (Table 1, Fig. 1). Since 1997, a number of stations in this depth range have been sampled periodically in order to follow the temporal and spatial succession of the benthic foraminiferal faunas.

The Bay of Biscay is a typical temperate mesooligotrophic environment with two annual bloom periods. The first one, in late winter or early spring, is associated with a shallowing of the mixed layer and with a strengthening of the rather shallow thermocline. Moreover, internal waves associated with strong spring neap tides cause nutrient injection into the photic zone and subsequent phytoplankton blooms at the shelf break in the northern part of the basin (Pingree et al., 1986). The second main bloom event, less marked than the first one, is commonly recorded in autumn, when the summer thermocline starts to be eroded by a deepening of mixing. The advantage of a study of benthic foraminiferal ecosystems in this mesotrophic-oligotrophic context is the fact that the oxygenation at the sediment-water interface is not seriously influenced by the seasonal variability of the organic matter input. The bottom water oxygen concentrations at the five stations are always relatively high (Table
1), and the seasonal monitoring of the oxygenation indicates that the concentrations do not vary significantly throughout the year (Anschutz et al., 1999; Hyacinthe et al., 2001). Therefore, ecosystem variability is probably caused mainly by changes in the organic flux reaching the ocean bottom. The downward organic flux varies with water depth and in response to the temporal and spatial oscillations of primary production in the surface waters. The five stations discussed in this paper were all sampled in late autumn-early winter (of the years 1997 and 1998; Table 1). The stations were selected in order to better understand the influence of the spatially variable organic matter flux reaching the ocean bottom on benthic foraminiferal faunas in an open slope setting. Our study focuses on the faunal density and composition and on foraminiferal microhabitats. The microhabitat is defined as the vertical distribution of a taxon in the first cms of the sediment, which is controlled by the composite action of all physical, chemical and biological processes (Corliss, 1985). Understanding the foraminiferal microhabitat is important, because it allows the precise trophic and oxic requirements of each species in the total live benthic foraminiferal assemblage to be known.

The main objectives of this paper are (1) to present and discuss variation of the foraminiferal density with increasing water depth and thus with calculated diminishing organic fluxes, (2) to better explain the compositional changes observed along the five stations bathymetrical/trophic transect, and (3) to explain the microhabitat changes in response to the trophic conditions at the sedimentwater interface.

Table 1

Water depth, sampling date, geographical position, bottom water oxygen and depth in the sediment of the zero oxygen level for the five stations used in this paper

\begin{tabular}{|c|c|c|c|c|c|c|c|}
\hline Station & Sample & Depth (m) & Date & Latitude & Longitude & Oxygenation $(\mathrm{ml} / \mathrm{l})$ & Zero $\mathrm{O}_{2}$ boundary $(\mathrm{mm})$ \\
\hline $\mathrm{D}$ & OB1D & 140 & October 1997 & $43^{\circ} 41^{\prime} 93 \mathrm{~N}$ & $1^{\circ} 34^{\prime} 10 \mathrm{~W}$ & 4.90 & 8 \\
\hline $\mathrm{B}$ & OB1B & 553 & October 1997 & $43^{\circ} 49^{\prime} 98 \mathrm{~N}$ & $2^{\circ} 23^{\prime} 04 \mathrm{~W}$ & 4.80 & 17 \\
\hline A & OB1A & 1012 & October 1997 & $44^{\circ} 09^{\prime} 08 \mathrm{~N}$ & $2^{\circ} 20^{\prime} 27 \mathrm{~W}$ & 4.36 & 20 \\
\hline $\mathrm{F}$ & $\mathrm{OB} 2 \mathrm{~F}$ & 1264 & Februar 1998 & $44^{\circ} 17^{\prime} 10 \mathrm{~N}$ & $2^{\circ} 44^{\prime} 95 \mathrm{~W}$ & 4.70 & 64 \\
\hline $\mathrm{H}$ & $\mathrm{OB} 5 \mathrm{H}$ & 1993 & October 1998 & $44^{\circ} 32^{\prime} 00 \mathrm{~N}$ & $2^{\circ} 37^{\prime} 00 \mathrm{~W}$ & 5.85 & 63 \\
\hline
\end{tabular}






Fig. 1. Study area, bathymetry and geographical position of the five stations.

\section{Study area, material and methods}

The Bay of Biscay is a semi-enclosed basin at the eastern side of the North Atlantic Ocean, bathed by rather homogeneous oceanic waters belonging to the North Atlantic thermohaline and geostrophic circulation and more precisely, to the North Atlantic Drift. The Bay of Biscay is bordered by the Irish shelf in the north, the Armorican and Aquitaine shelves in the east, and by the Iberian shelf in the south. The hydrographical structure is relatively well known (Ogawa and Tauzin, 1973). The patterns of the surface waters are strongly constrained by seasonal variations of the thermocline and the mixed layer. Below the surface waters ( $\leqslant 150 \mathrm{~m}$ depth), the Northern Atlantic Central Waters (NACW) are present down to $800 \mathrm{~m}$ depth. Between 800 and $1200 \mathrm{~m}$, a branch of Mediterranean outflow waters is present. In comparison with the surrounding water masses, these Mediterranean Waters (MW) are characterised by a high salinity (35.80-35.85, Le Floch, 1968). They have the lowest oxygen values for the Bay of Biscay $(3.8 \mathrm{ml} / 1$, Le Floch, $1968 ; 4.36 \mathrm{ml} / 1$, this paper). Below the MW,
Intermediate Atlantic Water and Polar Atlantic Water, both belonging to Northern Atlantic Deep Waters, fill the deepest parts of the basin. Within the Bay of Biscay, exchanges between the deeper parts and the coastal waters are rather insignificant, especially in spring and summer when a dome of cold and dense water on the continental shelves limits advection of sub-surface water (Ogawa and Tauzin, 1973).

The continental slope bordering the French shelf deepens gradually, and is interrupted by two large canyons (Cap Ferret Canyon, Cap Breton Canyon, Fig. 1). Vertical fluxes represent the main sedimentary component in open slope environments, whereas lateral advection may dominate sedimentary processes in the canyons (Heussner et al., 1999). The present paper concentrates on the open-slope environments, where the impact of laterally advected particles is supposed to be minimal, and where the linkage between surface water primary production, the vertical particle flux, and benthic life should be rather straightforward.

Few data are available on primary production in general or on the occurrences of bloom events in 
the Bay of Biscay. Tréguer et al. (1979) estimate a production between 0.4 and $1.9 \mathrm{~g} \mathrm{C} / \mathrm{m}^{2} /$ day for the spring bloom of 1973. Measurements of primary production in the central part of the Bay of Biscay during the autumn bloom of 1972 yielded values between 0.3 and $0.4 \mathrm{~g} \mathrm{C} / \mathrm{m}^{2} /$ day (Le Corre and Treguer, 1976). These values agree with recent data obtained in the Cap Ferret region during five ECOFER cruises: from 0.7 to $1.2 \mathrm{~g} \mathrm{C} / \mathrm{m}^{2} /$ day in spring (May 1990 and 1991) and $0.3 \mathrm{~g} \mathrm{C} / \mathrm{m}^{2} /$ day in October 1990 (Laborde et al., 1999). Laborde et al. (1999) estimate total annual primary production between 145 and $170 \mathrm{~g} \mathrm{C} / \mathrm{m}^{2} / \mathrm{yr}$. Generally, phytoplankton blooms in the Bay of Biscay are composed of diatoms (mainly Chaetoceros spp. and Nitzschia spp.) and coccolithophorids (Gephyrocapsa oceanica, Emiliania huxleyi; Tréguer et al., 1979; Fernandez et al., 1995). Fernandez (1990), however, shows that in the Central Cantabrian Sea (southern part of the Bay of Biscay) high chlorophyll- $a$ and primary production rates observed in March consist predominantly of microflagellates. Moreover, in the Celtic Sea, coccolithophore blooms (Emiliania huxleyi) may also occur during summer (Fernandez et al., 1993). Finally, Pingree et al. (1986) demonstrate a chlorophyll increase over the shelf break of the northern French shelf, due to internal and tidal waves; these rather exceptional and short bloom events occur during neap tides in spring.

In cases where the particle flux is mainly vertical, and where we have a quantitative estimate of the surface water primary production, we can use the formula proposed by Berger and Wefer (1990), and improved by Herguera (1992), to estimate the annual organic matter flux $J z$ that reaches the ocean bottom at different water depths:

$$
J z=(2 \sqrt{ } \mathrm{PP}(\mathrm{PP} / z))+((5 / \sqrt{ } \mathrm{PP})(\mathrm{PP} / \sqrt{ } z)),
$$

where $\mathrm{PP}$ is the primary production, in $\mathrm{g} \mathrm{C} / \mathrm{m}^{2} / \mathrm{yr}$, $z$ the water depth (in $\mathrm{m}$ ), $J z$ the organic flux at a water depth of $z$ meters, in $\mathrm{g} \mathrm{C} / \mathrm{m}^{2} / \mathrm{yr}$.

This formula differentiates between a labile component (first term), which is supposed to represent easily metabolisable organic detritus, and a more refractory component (second term), which decreases much more slowly with depth. We assume that benthic life will be affected mainly by the first term. When we use a mean primary production value of $150 \mathrm{~g} \mathrm{C} / \mathrm{m}^{2} / \mathrm{yr}$, for all stations, irrespective of water depth and distance from shore, and when we consider only the vertical transport of particles, the total exported flux at the shallowest station $(\mathrm{D}, 140 \mathrm{~m})$ can be estimated at $31.4 \mathrm{~g} \mathrm{C} / \mathrm{m}^{2} / \mathrm{yr}$, with a labile fraction of $26.6 \mathrm{~g} \mathrm{C} /$ $\mathrm{m}^{2} / \mathrm{yr}$. At the deepest station $(\mathrm{H}, 1993 \mathrm{~m})$ the estimated total exported flux is $3.2 \mathrm{~g} \mathrm{C} / \mathrm{m}^{2} / \mathrm{yr}$, with a labile component of $1.8 \mathrm{mg} \mathrm{C} / \mathrm{m}^{2} / \mathrm{yr}$. Estimated total flux values for the intermediate stations $\mathrm{B}$ $(553 \mathrm{~m}), \mathrm{A}(1012 \mathrm{~m})$ and $\mathrm{F}(1264 \mathrm{~m})$ are 9.2, 5.6 and $4.6 \mathrm{~g} \mathrm{C} / \mathrm{m}^{2} / \mathrm{yr}$, respectively; whereas the estimates for the labile component are 6.6, 3.6 and $2.9 \mathrm{~g} \mathrm{C} / \mathrm{m}^{2} / \mathrm{yr}$, respectively. These values suggest that the labile organic carbon flux, which should represent a readily available food source for the benthic fauna, is about 15 times higher in the shallowest station D than in the deepest station $\mathrm{H}$.

The five stations $\mathrm{D}, \mathrm{B}, \mathrm{A}, \mathrm{F}$, and $\mathrm{H}$ have been selected to compose a rather ideal SE-NW transect, ranging from the outer shelf to bathyal open slope environments (Fig. 1; Table 1). Station $\mathrm{D}$ is situated at $140 \mathrm{~m}$ depth, at the diffusive boundary between the surface waters and the NACW. According to Ogawa and Tauzin (1973), the NACW and the basal part of surface waters can be characterised by a seasonally constant temperature of $11.9^{\circ} \mathrm{C}$ and an invariant salinity of 35.60. The temperature and salinity of more superficial surface waters $(0-100 \mathrm{~m}$ depth) are strongly influenced by the seasonality of mixing, thermocline depth, and continental runoff. The sandy mud deposits found at station D are typical of the sedimentary conditions found in outer shelf environments.

Station B is positioned at $553 \mathrm{~m}$ depth. This site represents a mid-slope environment, isolated from possible lateral input of sediment (turbidites and slumps), which could occur in this depth range in areas closer to the two main canyon systems. Station B is positioned within the NACW, which has a salinity of 35.60 and a temperature of about $11^{\circ} \mathrm{C}$ (Ogawa and Tauzin, 1973). The sediment consists predominantly of fine-grained silty mud. 
Station A (1012 m depth) was selected for its strategic position inside the branch of Mediterranean outflow waters (MW). This water mass has a temperature of about $10.5^{\circ} \mathrm{C}$ and a salinity of 35.80 (Le Floch, 1968; Ogawa and Tauzin, 1973). Sediments at this depth are predominantly silty muds.

Station $\mathrm{F}$, at $1264 \mathrm{~m}$ deep, is positioned in the upper layers of Northern Atlantic Deep Waters (Intermediate Atlantic Waters), commonly characterising the bottom waters of the Celtic Sea $\left(8^{\circ} \mathrm{C}\right.$, 35.65, Ogawa and Tauzin, 1973). The sediments are silty muds.

Station H, at $1993 \mathrm{~m}$ depth, is typical for benthic environments found in the Northern Atlantic Deep Waters. Although this station is geographically rather close to the Cap Ferret Canyon, it is still an open slope environment with muddy sediments. The water temperature is about $4{ }^{\circ} \mathrm{C}$ and the salinity is about 35.00 (Ogawa and Tauzin, 1973).

All cores were sampled with a classic Barnett multi-tube corer (Barnett et al., 1984). Each tube has a surface area of about $72 \mathrm{~cm}^{2}$, and accordingly, all our faunal density data are standardised for this surface area. The multi-corer allows sampling of the first $\mathrm{dm}$ of the sediment, the overlying bottom waters, and an undisturbed sediment-water interface. Free waters were collected immediately after core recovery for dissolved $\mathrm{O}_{2}$ measurements by the Winkler titration method (Strickland and Parsons, 1972). Profiles of pore water $\mathrm{O}_{2}$ were obtained on board with a cathode-type mini-electrode (Revsbech and Jørgensen, 1986; Helder and Bakker, 1985; Revsbech, 1983). The temperature was maintained by using an insulating device. This operation was completed in duplicate within $30 \mathrm{~min}$ after core recovery. Subsequently, the core used for $\mathrm{O}_{2}$ profiling was sliced in thin horizontal sections (every $0.5 \mathrm{~cm}$ for the top $2 \mathrm{~cm}, 1$ or $2 \mathrm{~cm}$ below) within $1 \frac{1}{2} \mathrm{~h}$. For every level a sub-sample was immediately sealed in a pre-weighed vial and frozen under inert atmosphere $\left(\mathrm{N}_{2}\right)$ for further analyses of porosity and chemistry of the solid fraction. Another sub-sample was centrifuged under $\mathrm{N}_{2}$ at $5000 \mathrm{rpm}$ for $20 \mathrm{~min}$ for collection pore waters. Two aliquots of water were filtered
$(0.2 \mu \mathrm{m})$ and frozen at $-25^{\circ} \mathrm{C}$ for nutrient analyses. In the laboratory, porosity was determined by comparison of the weights of wet and freezedried sediment. Interstitial water compounds were analysed by techniques adapted for small volumes (Anschutz et al., 1999; Hyacinthe et al., 2001). Nitrate and nitrite were measured by flow injection analysis (FIA) according to Anderson (1979). Sulphate was measured by a nephelometric method (Stookey, 1970).

For faunal analysis, one entire core was sliced horizontally for each station; every $0.25 \mathrm{~cm}$ for the first $\mathrm{cm}$ of sediment, every half $\mathrm{cm}$ between 1 and $4 \mathrm{~cm}$ depth, and every $\mathrm{cm}$ between 4 and $10 \mathrm{~cm}$. Exceptionally, for station A, the core was cut into $0.5 \mathrm{~cm}$ slices between 1 and $4.5 \mathrm{~cm}$, and, for station $\mathrm{D}$, the first $\mathrm{cm}$ was cut into 3 slices $(0-0.35,0.35-$ 0.75 and $0.75-1.0 \mathrm{~cm}$ ). Sediments were stored in $500 \mathrm{~cm}^{3}$ bottles, which were filled with $95 \%$ ethanol containing $1 \mathrm{~g} / 1$ Rose Bengal stain. Rose Bengal staining is commonly used to identify live foraminifera (Walton, 1952). All samples were gently shaken for several minutes in order to get a homogeneous mixture. In the laboratory, they were sieved through 63 and $150 \mu \mathrm{m}$ mesh screens, and the sieve residues were stored in $95 \%$ ethanol. Stained foraminifera belonging to the $150 \mu \mathrm{m}$ fraction were sorted from wet samples, and stored in Chapman slides. The $63-150 \mu \mathrm{m}$ fraction was preserved for future studies. The interpretation of staining of benthic foraminifera is rather subjective. One problem of this technique is the fact that Rose Bengal may stain the protoplasm of dead foraminifera, which may be relatively well preserved for a considerable period of time under the anoxic conditions that generally prevail deep in the sediment (Bernhard, 1988; Corliss and Emerson, 1990). As a consequence, a strict application of the staining criteria is most times easy in superficial samples, but may become more critical in the deeper levels. In all cases, we applied our staining criteria (all chambers except the last one stained brightly pink) very strictly, and compared doubtful individuals with perfectly stained individuals of the same species found in superficial sediment layers. Non-transparent agglutinated and miliolid taxa were broken on many occasions for inspection of the interior of the test. We tried to 
identify most of live foraminifera at specific level. We used taxonomic references which are presented in Appendix A. Fragments of branchlike agglutinating foraminifera (such as Hyperammina) and Glomospira charoides and Glomospira gordialis (which, because of the orange-reddish colour of their test are very difficult to confirm as living), were not included in the quantitative analyses.

In order to describe the vertical distribution of the total faunas or individual taxa, we use the average living depth (ALD, Jorissen et al., 1995), which allows a rapid description of the microhabitat patterns. The ALD is calculated with the following formula:

$$
\operatorname{ALD}_{x}=\sum_{i=0, x}\left(n_{i} D_{i}\right) / N
$$

where $x$ is the lower boundary of deepest sample, $n_{i}$ the number of individuals in interval $i, D_{i}$ the midpoint of sample interval $i, N$ the total number of individuals for all levels.

For all stations, $\mathrm{ALD}_{10}$ was calculated for the whole fauna as well as for individual taxa, on the basis of the numbers of stained individuals found in the successive sediment slices. Isolated individuals separated from the main population by more than $1 \mathrm{~cm}$ of "sterile" sediment (without live individuals of the studied taxa) were not integrated in the calculations of the $A L D_{10}$. We suppose that such isolated individuals had been transported downward (outside their normal microhabitat) by bioturbation, or correspond to dead organisms that have been counted erroneously. In the data sheets (Tables $2 \mathrm{a}-\mathrm{e})$, the latter individuals are indicated in brackets.

After the first classification with four main microhabitats proposed by Corliss and Chen (1988), it was argued that only species living on elevated substrates can be considered as "epifaunal" (Buzas et al., 1993). Therefore, in the soft bottom communities described in this study, we recognise only three different groups: shallow infaunal, intermediate infaunal and deep infaunal species.

\section{Results}

The total density of the live foraminiferal fauna were determined by integrating the numbers of live individuals picked in all levels from 0 to $10 \mathrm{~cm}$ depth. It is expressed as the number of live foraminifera found in and below a $72 \mathrm{~cm}^{2}$ surface area (Fig. 2). Concerning vertical profiles, specific foraminiferal densities were normalised for each layer to a $50 \mathrm{~cm}^{3}$ sediment volume; we generally regrouped the four uppermost slices of each core into two $0.5 \mathrm{~cm}$ thick samples. The percentages of the various taxa were calculated on the basis of the non normalised densities.

Fig. 2, which presents the foraminiferal densities for the five stations, shows a clear negative correlation between the foraminiferal density and water depth. The shallowest station $\mathrm{D}$, with about 2000 live individuals collected, presents a maximum value, whereas stations $\mathrm{H}$ and $\mathrm{F}$, with 179 and 122 individuals, respectively, are much poorer.

At all five stations, perforate foraminifera form the main component of the benthic foraminiferal faunas (75-90\%; Fig. 2). At station B, they compose almost $90 \%$ of the total fauna. Agglutinated taxa account for $10-20 \%$ of the total fauna, whereas miliolids are rare in all stations (between $0.3 \%$ and $8.2 \%$ ). The latter group tends to be richer on the middle and lower slope (between $4.2 \%$ and $8.2 \%$ ) than on the outer shelf and upper slope, where they are almost absent.

Species diversity is highest at station B where about 50 species are found. Station D, with 36 species, is slightly less diverse. Station A contains 41 species; at stations $\mathrm{F}$ and $\mathrm{H}$, where agglutinated and porcellaneous taxa represent more than half of all species, 25 and 28 species are found, respectively.

Bottom waters at station D $(140 \mathrm{~m})$ have an oxygen concentration of $4.9 \mathrm{ml} / 1$. Within the sediment, there is a rapid decrease, until anoxic conditions are reached at $0.8 \mathrm{~cm}$ depth (Fig. 3a). The nitrate + nitrite profile shows a sharp decrease in the first $\mathrm{mm}$ of the sediment, suggesting that also the zone of nitrate reduction is extremely close to the sediment-water interface. Sulphate reduction is chemically detected below $5 \mathrm{~cm}$ depth, and corresponds to an increase of particulate sulphur 
and a decrease of dissolved sulphate. This is the only station where sulphate reduction is significant. Station D, which has the highest foraminiferal density of all five stations (1989 individuals/ $72 \mathrm{~cm}^{2}$ ), presents a faunal assemblage that is strongly concentrated in the uppermost $\mathrm{cm}$ of the sediment (Fig. 3b). The highest density is recorded in the superficial sample $(0.00-0.35 \mathrm{~cm})$, where a value of 1400 live individuals $/ 50 \mathrm{~cm}^{3}$ has been calculated. This value falls abruptly to about 350 individuals $/ 50 \mathrm{~cm}^{3}$ in the $0.75-1.0 \mathrm{~cm}$ level, where the oxygen concentration is already close to zero. About 350 live foraminifera $/ 50 \mathrm{~cm}^{3}$ can still be noted in the $1.0-1.5 \mathrm{~cm}$ level, in a completely anoxic environment. The $\mathrm{ALD}_{10}$ of the total live fauna is $1.1 \mathrm{~cm}$. The fauna is dominated by Chilostomella oolina (30.2\%), Valvulineria bradyana $(17.0 \%)$, Clavulina cylindrica $(15.0 \%)$, Nonion scaphum (13.0\%), Bolivina subaenariensis $(3.1 \%)$, Hyalinea balthica $(2.7 \%)$ and Bulimina marginata $(2.3 \%)$ (Table $2 \mathrm{a})$. V. bradyana $\left(\mathrm{ALD}_{10}=0.4 \mathrm{~cm}\right)$, C. cylindrica $\left(\mathrm{ALD}_{10}=0.4 \mathrm{~cm}\right)$, and $C$. oolina $\left(\mathrm{ALD}_{10}=1.2 \mathrm{~cm}\right)$ are the dominant taxa in the well-oxygenated first half $\mathrm{cm}$ (Fig. 3c). They are accompanied there by less frequent species such as Bolivina alata, B. subaenariensis, Uvigerina peregrina and Rectuvigerina phlegeri. C. oolina $\left(\mathrm{ALD}_{10}=1.2 \mathrm{~cm}\right)$, and $N$. scaphum $\left(\mathrm{ALD}_{10}=\right.$ $1.8 \mathrm{~cm}$ ) dominate the faunas in the anoxic zone from 1 to $3 \mathrm{~cm}$ depth. Some rare individuals of Bulimina aculeata and B. marginata are found in the totally anoxic environments below $3 \mathrm{~cm}$ depth.

At station B (water depth $553 \mathrm{~m}$ ), the bottom water oxygen concentration is $4.8 \mathrm{ml} / 1$. The zero oxygen level is found at about $2 \mathrm{~cm}$ depth (Fig. 4a). Well-oxygenated conditions prevail in the first half $\mathrm{cm}$. A downward diffusive zone of nitrate and nitrite extends from 0.25 to $2.25 \mathrm{~cm}$ depth. Fig. 4b shows that maximal densities (between 800 and 650 individuals $/ 50 \mathrm{~cm}^{3}$ ) are found in the top first $\mathrm{cm}$. Faunal numbers drastically drop to about 40 individuals $/ 0.5 \mathrm{~cm}$ level at $1.75 \mathrm{~cm}$ depth. The $\mathrm{ALD}_{10}$ of the total live fauna is $0.8 \mathrm{~cm}$. The benthic foraminiferal fauna is strongly dominated by Uvigerina mediterranea $(33.8 \%)$, Uvigerina peregrina $(21.6 \%)$, Melonis barleeanus (9.0\%), Globobulimina affinis (6.0\%) and Cibicidoides pachydermus (4.7\%) (Table 2b).
$U$. mediterranea $\left(\mathrm{ALD}_{10}=0.6 \mathrm{~cm}\right)$, accompanied by $C$. pachydermus $\left(\mathrm{ALD}_{10}=0.6 \mathrm{~cm}\right)$ and $U$. peregrina $\left(\mathrm{ALD}_{10}=0.8 \mathrm{~cm}\right)$, dominate the superficial sediments (Fig. 4c). Slightly deeper, between 0.5 and $1.5 \mathrm{~cm}$ depth, $U$. peregrina $\left(\mathrm{ALD}_{10}=\right.$ $0.8 \mathrm{~cm})$ and $M$. barleeanus $\left(\mathrm{ALD}_{10}=0.8 \mathrm{~cm}\right)$ show maximum frequencies. G. affinis $\left(\mathrm{ALD}_{10}=2.4 \mathrm{~cm}\right)$ thrives preferentially in the deeper part of the core. It increases below $1.0 \mathrm{~cm}$ depth and becomes the only remaining dominant taxon below 2.25 and $6.5 \mathrm{~cm}$ depth.

At station A (water depth $1012 \mathrm{~m}$ ), the oxygen concentration is $4.36 \mathrm{ml} / 1$. The zero oxygen level is found at about $2.0 \mathrm{~cm}$ depth, whereas a nitrate + nitrite downward diffusive zone extends from 0.75 to $3.5 \mathrm{~cm}$ depth (Fig. 5a). Fig. 5b depicts a bimodal distribution of the benthic foraminiferal fauna. A first density maximum (180 individuals $\left./ 50 \mathrm{~cm}^{3}\right)$ is recorded around $1 \mathrm{~cm}$ depth, a second one (40 individuals $/ 50 \mathrm{~cm}^{3}$ ) occurs deeper in the sediment, at about $6 \mathrm{~cm}$ depth. The $\mathrm{ALD}_{10}$ of the total live fauna is $2.4 \mathrm{~cm}$. Globobulimina affinis $(28.8 \%)$, Uvigerina peregrina (17.9\%), Uvigerina mediterranea $(9.5 \%)$, Hoeglundina elegans (5.5\%), Nuttallides umboniferus (5.3\%), Cibicidoides pachydermus $(1.9 \%)$ and Melonis barleeanus $(1.3 \%)$ are the dominant taxa of station A (Table 2c). $N$. umboniferus $\left(\mathrm{ALD}_{10}=0.4 \mathrm{~cm}\right), U$. mediterranea $\left(\mathrm{ALD}_{10}=0.6 \mathrm{~cm}\right), \quad C$. pachydermus $\left(\mathrm{ALD}_{10}=\right.$ $0.3 \mathrm{~cm})$ and $H$. elegans $\left(\mathrm{ALD}_{10}=0.8 \mathrm{~cm}\right)$ constitute a rich superficial fauna, which is present in the well-oxygenated first $\mathrm{cm}$ (Fig. 5c). U. peregrina $\left(\mathrm{ALD}_{10}=1.1 \mathrm{~cm}\right)$, accompanied by $M$. barleeanus $\left(\mathrm{ALD}_{10}=1.7 \mathrm{~cm}\right)$, occupies slightly deeper layers and is the dominant component of the shallowest density peak. G. affinis $\left(\mathrm{ALD}_{10}=5.3 \mathrm{~cm}\right)$, accompanied by some individuals of Glandulina ovula $\left(\mathrm{ALD}_{10}=5.0 \mathrm{~cm}\right)$ and $C$. oolina $\left(\mathrm{ALD}_{10}=5.5 \mathrm{~cm}\right)$, dominates the rich stained fauna below $2 \mathrm{~cm}$ depth.

The oxygen concentration of the bottom waters at station F (water depth $1264 \mathrm{~m}$ ) is $4.70 \mathrm{ml} / 1$. Within the sediment, the zero oxygen level is reached at $6.4 \mathrm{~cm}$ depth (Fig. 6a). The dissolved oxygen curve shows some minor oscillations that may be the result of microenvironments caused by burrows. A nitrate diffusive gradient extends from 4.5 to $7.5 \mathrm{~cm}$ depth. The benthic foraminiferal 
Table 2

Benthic foraminiferal census data for stations D, B, A, F and H. N.B. Numbers are not standardized for a sediment volume

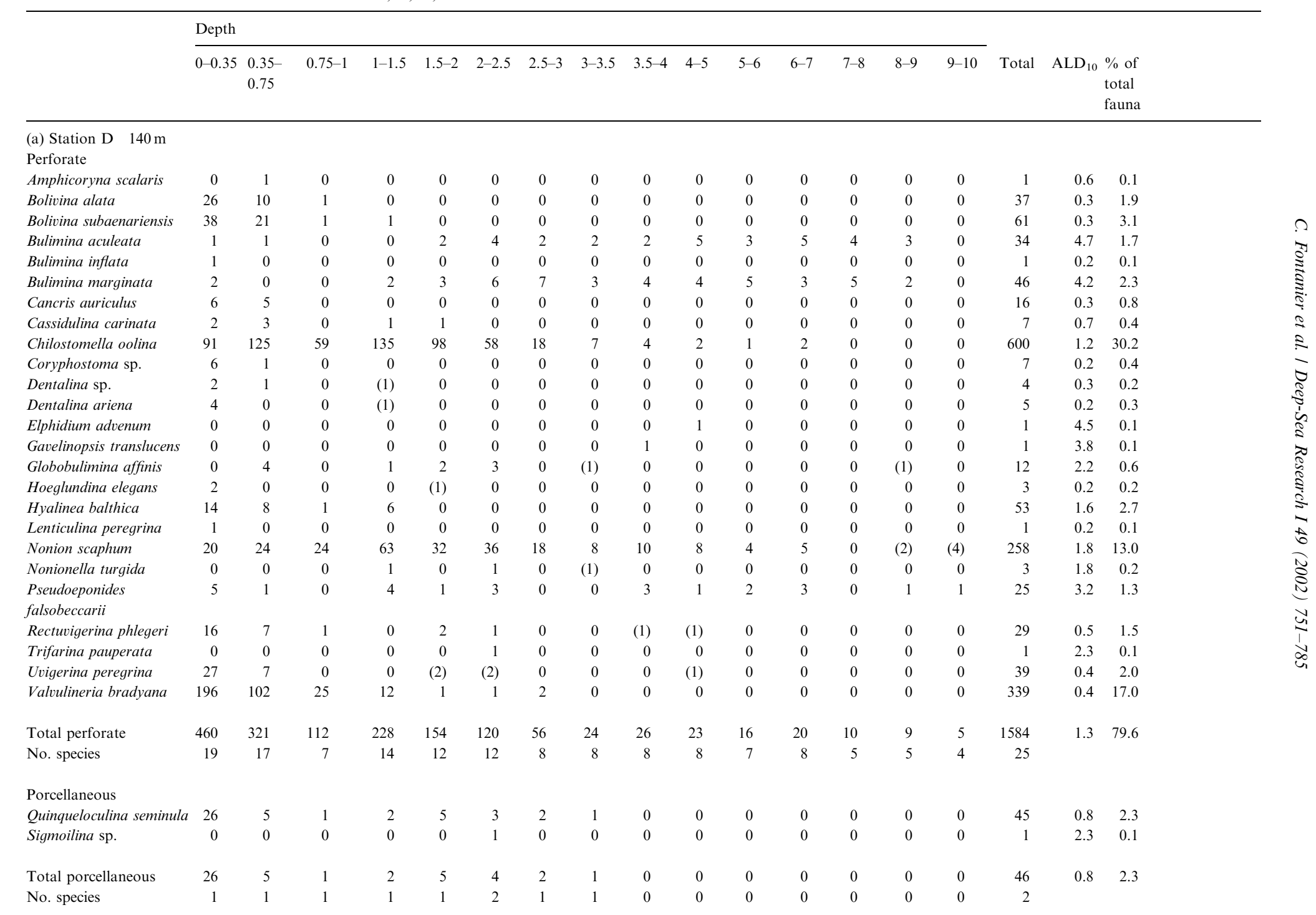


Non fossilizing agglutinated

$\begin{array}{lll}\text { Clavulina cylindrica } & 208 & 60\end{array}$

Eggerella sp.

Haplophragmoides sp.

Nouria polymorphinoides $11 \quad 3$

Reophax sp.

Reophax ampullacea

Reophax scorpiurus

Total agglutinated

No. species

Fossilizing agglutinated

Textularia agglutinan

Textularia sagittula

Total agglutinated

No. species

Total live foraminifera

No. species

\begin{tabular}{|c|c|c|c|c|c|c|c|c|c|c|c|c|c|c|c|c|}
\hline 30 & 29 & 21 & 17 & 17 & 9 & 9 & 8 & 8 & 7 & 8 & 5 & 5 & 4 & 36 & & \\
\hline Depth & & & & & & & & & & & & & & & & \\
\hline $0-0.25$ & $0.25-0.5 \quad 0.5-0.75$ & $0.75-1$ & $1-1.5$ & $1.5-2$ & $2-2.5$ & $2.5-3$ & $3-3.5$ & $3.5-4$ & $4-5$ & $5-6$ & $6-7$ & $7-8$ & $8-9$ & $9-10$ & Total & $\begin{array}{r}\operatorname{ALD}_{10} \% \text { of } \\
\text { total } \\
\text { faun }\end{array}$ \\
\hline
\end{tabular}

(b) Station B $553 \mathrm{~m}$

Perforate

Indet

Amphicoryna scalaris

Bolivina alata

Bolivinita quadrilatera

Bulimina marginata

Chilostomella oolina

Cibicides lobatulus

Cibicides wuellerstorfi

Cibicidoides pachydermus 26

Dentalina sp.

Elphidium sp.

Gavelinopsis translucens

Glandulina ovula

Globobulimina affinis

Gyroidina altiformis

Gyroidina orbicularis

Hyalinea balthica

Lenticulina gibba

$\begin{array}{rrrrrrrrrrrrrrr}11 & 4 & 4 & 0 & 0 & (1) & (1) & 0 & 0 & 0 & 0 & 0 & 298 & 0.4 & 15.0 \\ 4 & 0 & (6) & 0 & 0 & 0 & (1) & 0 & 0 & 0 & 0 & 0 & 18 & 0.5 & 0.9 \\ 1 & 0 & 0 & 0 & 0 & 0 & 0 & 0 & 0 & 0 & 0 & 0 & 8 & 0.4 & 0.4 \\ 1 & 0 & 0 & 0 & 0 & 0 & 0 & 0 & 0 & 0 & 0 & 0 & 16 & 0.4 & 0.6 \\ 0 & 0 & 0 & 0 & 0 & 0 & 0 & 0 & 0 & 0 & 0 & 0 & 6 & 0.3 & 0.3 \\ 1 & 1 & 0 & 0 & 0 & 0 & 0 & 0 & 0 & 0 & 0 & 0 & 2 & 1.5 & 0.1 \\ 0 & 0 & 0 & 0 & 0 & 0 & 0 & 0 & 0 & 0 & 0 & 0 & 1 & 0.2 & 0.1\end{array}$

gibba $\quad 0$

0
1
1
0
0
0
1
1
6
0
0
0
0
1
2
0
0
0

0
0
2
0
1
2
0
0
3
0

$\begin{array}{rrrrrrr}0 & 0 & 0 & 0 & 0 & 0 & 0 \\ 1 & 1 & 0 & 0 & 0 & 0 & 0 \\ 2 & 1 & 0 & 0 & 0 & 0 & 0 \\ 0 & 0 & 0 & 0 & 0 & (1) & 0 \\ 1 & 3 & 1 & 4 & 0 & 0 & 0 \\ 0 & 0 & 0 & 0 & 1 & 0 & 0 \\ 0 & 0 & 0 & 0 & 0 & 0 & 0 \\ 0 & 0 & 0 & 0 & 0 & 0 & 0 \\ 3 & 6 & 1 & 2 & 0 & 0 & 0 \\ 0 & 0 & 0 & 0 & 0 & 0 & 0 \\ 0 & 0 & 0 & 0 & 0 & 0 & 0 \\ 0 & 0 & 0 & 0 & 0 & 0 & 0 \\ 0 & 0 & 0 & 0 & 0 & 0 & 0 \\ 19 & 6 & 13 & 16 & 10 & 6 & 3 \\ 0 & (1) & 0 & 0 & 0 & 0 & 0 \\ 0 & 0 & 0 & 0 & 0 & 0 & 0 \\ 0 & 0 & (1) & (1) & (1) & 0 & 0 \\ 0 & 0 & 0 & 0 & 0 & 0 & 0\end{array}$

$\begin{array}{ll}0 & 0 \\ 0 & 0 \\ 0 & 0 \\ 0 & 0 \\ 0 & 0 \\ 0 & 0 \\ 0 & 0 \\ 0 & 0 \\ 0 & 0 \\ 0 & 0 \\ 0 & 0 \\ 0 & 0 \\ 0 & 0 \\ 3 & 0 \\ 0 & 0 \\ 0 & 0 \\ 0 & 0 \\ 0 & 0\end{array}$

0
0
0
0
0
0
0
0
0
0
0
0
0
0
0
0
0
0


Depth

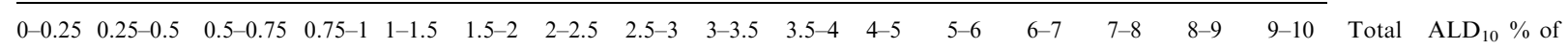

total

\begin{tabular}{|c|c|c|c|c|c|c|c|c|c|c|c|c|c|c|c|c|c|c|c|}
\hline Lenticulina peregrina & 4 & 3 & 0 & 0 & 0 & 0 & 0 & 0 & 0 & 0 & 0 & 0 & 0 & 0 & 0 & 0 & 7 & 0.2 & 0.5 \\
\hline Lenticulina rotulata & 2 & 0 & 0 & 1 & 0 & 0 & 0 & 0 & 0 & 0 & 0 & 0 & 0 & 0 & 0 & 0 & 3 & 0.4 & 0.2 \\
\hline Melonis barleeanus & 14 & 33 & 27 & 23 & 14 & 4 & 1 & 1 & 3 & 1 & 0 & 0 & 0 & 0 & 0 & 0 & 121 & 0.8 & 9.0 \\
\hline Nodosaria sp. & 0 & 0 & 1 & 0 & 0 & 0 & 0 & 0 & 0 & 0 & 0 & 0 & 0 & 0 & 0 & 0 & 1 & 0.6 & 0.1 \\
\hline Nuttallides umboniferus & 2 & 2 & 1 & 0 & 0 & 0 & 0 & 0 & 0 & 0 & 0 & (1) & 0 & 0 & 0 & 0 & 6 & 0.3 & 0.4 \\
\hline Pseudoeponides & 0 & 0 & 0 & 0 & 0 & 0 & 0 & 0 & 1 & 0 & 0 & 0 & 0 & 0 & 0 & 0 & 1 & 3.3 & 0.1 \\
\hline \multicolumn{20}{|l|}{ falsobeccarii } \\
\hline Robertinoides sp. & 3 & 0 & 0 & 0 & 0 & 0 & 0 & 0 & 0 & 0 & 0 & 0 & 0 & 0 & 0 & 0 & 3 & 0.1 & 0.2 \\
\hline Siphogenerina sp. & 13 & 7 & 4 & 1 & 0 & 2 & 1 & 1 & 1 & 0 & 0 & 0 & 0 & 0 & 0 & 0 & 30 & 0.6 & 2.2 \\
\hline Trifarina angulosa & 2 & 0 & 1 & 0 & 0 & 0 & 0 & 0 & 0 & 0 & 0 & 0 & 0 & 0 & 0 & 0 & 3 & 0.3 & 0.2 \\
\hline Uvigerina mediterranea & 119 & 123 & 108 & 54 & 20 & 14 & 4 & 2 & 0 & (1) & 0 & (1) & 0 & 0 & 0 & 0 & 454 & 0.6 & 33.8 \\
\hline Uvigerina peregrina & 30 & 43 & 83 & 78 & 43 & 7 & 3 & 2 & 0 & 0 & 0 & 0 & 0 & (1) & 0 & 0 & 290 & 0.8 & 21.6 \\
\hline Uvigerina proboscidea & 1 & 0 & 0 & 0 & 0 & 0 & 0 & 0 & 0 & 0 & 0 & 0 & 0 & 0 & 0 & 0 & 1 & 0.1 & 0.1 \\
\hline Total perforate & 246 & 238 & 243 & 187 & 134 & 48 & 29 & 38 & 19 & 8 & 3 & 6 & 0 & 1 & 1 & 0 & 1201 & 0.8 & 89.3 \\
\hline No. species & 21 & 12 & 15 & 13 & 9 & 11 & 9 & 10 & 7 & 4 & 1 & 4 & 0 & 1 & 1 & 0 & 33 & & \\
\hline \multicolumn{20}{|l|}{ Porcellaneous } \\
\hline Cruciloculina sp. & 1 & 0 & 0 & 0 & 0 & 0 & 0 & 0 & 0 & 0 & 0 & 0 & 0 & 0 & 0 & 0 & 1 & 0.1 & 0.1 \\
\hline Pyrgo depressa & 1 & 0 & 0 & 0 & 0 & 0 & 0 & 0 & 0 & 0 & 0 & 0 & 0 & 0 & 0 & 0 & 1 & 0.1 & 0.1 \\
\hline Sigmoilina sp. & 1 & 0 & 0 & 0 & 0 & 0 & 0 & 0 & 0 & 0 & 0 & 0 & 0 & 0 & 0 & 0 & 1 & 0.1 & 0.1 \\
\hline Total porcellaneous & 4 & 0 & 0 & 0 & 0 & 0 & 0 & 0 & 0 & 0 & 0 & 0 & 0 & 0 & 0 & 0 & 4 & 0.1 & 0.3 \\
\hline No. species & 4 & 0 & 0 & 0 & 0 & 0 & 0 & 0 & 0 & 0 & 0 & 0 & 0 & 0 & 0 & 0 & 4 & & \\
\hline
\end{tabular}

Ammolagena sp.

Ammoscalaria sp.

Clavulina cylindrica

Cribrostomoides

subglobosum

Cyclammina cancellala

Eggerella sp.

Haplophragmoides $\mathrm{sp}$.

Reophax sp.

Trochammina inflata

$\begin{array}{lll}\text { Total agglutinated } & 36 & 36 \\ \text { No. species } & 10 & 14\end{array}$

$\begin{array}{lll}\text { Total agglutinated } & 36 & 36 \\ \text { No. species } & 10 & 1\end{array}$

1
0
0
8

1
1
1
4

6

$0 \quad 0$

3

0

0

0
0
0
0

$\begin{array}{ll}0 & 0 \\ 0 & 0 \\ 0 & \\ 0 & 0\end{array}$

0
0
0
0

(1) (1) 0

0

$\begin{array}{ll}0 & 0 \\ 0 & 0 \\ 0 & 0 \\ 0 & 0 \\ 0 & 0\end{array}$

0
0
0

5
1
0
21

$\begin{array}{rr}0 & 2 \\ 21 & 19\end{array}$

6 
Fossilizing agglutinated

Bigenerina nodosaria

Pseudoclavulina crustata

Siphotextularia affinis

Total agglutinated

No. species

$\begin{array}{lllllllll}\text { Total live foraminifera } & 297 & 280 & 263 & 200 & 144 & 53 & 32 & 38\end{array}$

No. species

Glomospira spp

$35 \quad 26 \quad 26$

Arborescent indet

$\begin{array}{lllllll}1 & 4 & 6 & 4 & 11 & 10 & 11\end{array}$

Depth

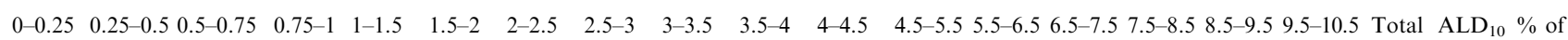

fauna

(c) Station A $1012 \mathrm{~m}$

\section{Perforate}

Bulimina inflata

Bulimina marginata

Chilostomella oolina

Cibicidoides pachydermus

Glandulina ovula

Globobulimina affinis

Gyroidina altiformis

Gyroidina orbicularis

Hoeglundina elegans

Lenticulina sp.

Melonis barleeanus

Nuttallides umboniferus

Pullenia sp.

Siphogenerina sp.

Rosalina sp.

Uvigerina mediterranea

Uvigerina peregrina

Total perforate

No. species

Porcellaneous

Biloculinella irregularis

Cyclogyra sp.

Pyrgo depressa

\begin{tabular}{|c|c|c|c|c|c|c|}
\hline 1 & 1 & 0 & 0 & 0 & 0 & 0 \\
\hline 0 & 0 & 0 & 0 & 0 & 0 & 0 \\
\hline 0 & 0 & 0 & 0 & 0 & 0 & 0 \\
\hline 4 & 2 & 3 & 0 & 0 & 0 & 0 \\
\hline 0 & 0 & 1 & 1 & 0 & 0 & 0 \\
\hline 0 & 0 & 0 & 0 & 0 & 0 & 3 \\
\hline 1 & 0 & 2 & 0 & 0 & 0 & 0 \\
\hline 0 & 0 & 0 & 0 & 2 & 0 & (1) \\
\hline 4 & 4 & 4 & 6 & 7 & 1 & 0 \\
\hline 0 & 0 & 1 & 0 & 1 & 0 & 0 \\
\hline 0 & 0 & 0 & 0 & 3 & 1 & 2 \\
\hline 8 & 8 & 6 & 2 & 1 & 0 & 0 \\
\hline 0 & 0 & 0 & 0 & 0 & 0 & 1 \\
\hline 0 & 0 & 0 & 0 & 0 & 0 & 0 \\
\hline 1 & 0 & 0 & 0 & 0 & 0 & 0 \\
\hline 5 & 12 & 12 & 12 & 4 & 0 & 0 \\
\hline 2 & 1 & 10 & 22 & 37 & 13 & 0 \\
\hline
\end{tabular}

(1)

$\begin{array}{rrr}0 & 0 & 0 \\ 0 & 0 & 0 \\ 0 & 0 & 1 \\ 0 & 0 & 0 \\ 1 & 2 & 2 \\ 8 & 10 & 10 \\ 0 & 0 & 0 \\ 0 & 0 & 0 \\ 0 & 0 & 0 \\ 0 & 0 & 0 \\ 0 & 0 & 0 \\ 0 & 0 & 0 \\ 0 & 0 & 0 \\ 0 & 1 & 0 \\ 0 & 0 & 0 \\ 0 & 0 & 0 \\ 0 & 0 & 0\end{array}$

$\begin{array}{rrrrr}43 & 55 & 15 & 7 & 13 \\ 5 & 7 & 3 & 4 & 1 \\ & & & & \\ 1 & 0 & 0 & 0 & 0 \\ 1 & 0 & (1) & 0 & 0 \\ 0 & 0 & 0 & 0 & 0\end{array}$

$\begin{array}{ll}3 & 10 \\ 1 & 3 \\ & \\ 0 & 0 \\ 0 & 0 \\ 0 & 0\end{array}$

$\begin{array}{rrr}0 & 13 & 13 \\ 3 & 3 & -1 \\ 0 & 0 & \\ 0 & 0 & 0 \\ 0 & 0 & \end{array}$

$\begin{array}{rrrrrrrrr}0 & 0 & 0 & 0 & 0 & 0 & 2 & 0.2 & 0.4 \\ 1 & 0 & 0 & 0 & 0 & 0 & 1 & 4.8 & 0.2 \\ 1 & 1 & 1 & 0 & 0 & 0 & 4 & 5.5 & 0.8 \\ 0 & 0 & 0 & 0 & 0 & 0 & 9 & 0.3 & 1.9 \\ 0 & 1 & 1 & 2 & 0 & 1 & 12 & 5.0 & 2.5 \\ 17 & 44 & 27 & 5 & 0 & 0 & 137 & 5.3 & 28.8 \\ 0 & 0 & 0 & 0 & 0 & 0 & 3 & 0.5 & 0.6 \\ 0 & 0 & 0 & 0 & 0 & 0 & 4 & 1.3 & 0.8 \\ 0 & 0 & 0 & 0 & 0 & 0 & 26 & 0.8 & 5.5 \\ 0 & 0 & 0 & 0 & 0 & 0 & 2 & 0.9 & 0.4 \\ 0 & 0 & 0 & 0 & 0 & 0 & 6 & 1.7 & 1.3 \\ 0 & 0 & 0 & 0 & 0 & 0 & 25 & 0.4 & 5.3 \\ 0 & 0 & 0 & 0 & 0 & 0 & 1 & 2.3 & 0.2 \\ 0 & 1 & 0 & 0 & 0 & 0 & 2 & 4.9 & 0.4 \\ 0 & 0 & 0 & 0 & 0 & 0 & 1 & 0.1 & 0.2 \\ 0 & 0 & 0 & 0 & 0 & 0 & 45 & 0.6 & 9.5 \\ 0 & 0 & 0 & 0 & 0 & 0 & 85 & 1.1 & 17.9 \\ & & & & & & & & \\ 19 & 47 & 29 & 7 & 0 & 1 & 365 & 2.7 & 76.7 \\ 3 & 4 & 3 & 2 & 0 & 1 & 20 & & \\ & & & & & & & & \\ 0 & 0 & 0 & 0 & 0 & 0 & 1 & 0.9 & 0.2 \\ 0 & 0 & 0 & 0 & 0 & 0 & 4 & 0.3 & 0.8 \\ 0 & 0 & 0 & 0 & 0 & 0 & 2 & 0.6 & 0.4\end{array}$


Depth

$0-0.25 \quad 0.25-0.5 \quad 0.5-0.75 \quad 0.75-1 \quad 1-1.5 \quad 1.5-2 \quad 2-2.5 \quad 2.5-3 \quad 3-3.5 \quad 3.5-4 \quad 4-4.5 \quad 4.5-5.5 \quad 5.5-6.5 \quad 6.5-7.5 \quad 7.5-8.5 \quad 8.5-9.5 \quad 9.5-10.5 \quad$ Total $\quad$ ALD $10 \%$ of

total

\begin{tabular}{|c|c|c|c|c|c|c|c|c|c|c|c|c|c|c|c|c|c|c|c|c|}
\hline & & & & & & & & & & & & & & & & & & & & fauna \\
\hline Pyrgo subsphaerica & 0 & 0 & 1 & 1 & 2 & 0 & 0 & 0 & 0 & 0 & 0 & 0 & 0 & 0 & 0 & 0 & 0 & 4 & 1.0 & 0.8 \\
\hline Pyrgoella sphaera & 2 & 1 & 0 & 0 & 0 & 0 & (1) & 0 & 0 & 0 & 0 & 0 & 0 & 0 & 0 & 0 & 0 & 4 & 0.2 & 0.8 \\
\hline Quinqueloculina seminula & 1 & 0 & 0 & 0 & 0 & 0 & 0 & 0 & 0 & 0 & 0 & 0 & 0 & 0 & 0 & 0 & 0 & 1 & 0.1 & 0.2 \\
\hline Quinqueloculina sp. & 0 & 0 & 1 & 0 & 0 & (2) & 0 & 0 & 0 & 0 & 0 & 0 & 0 & 0 & 0 & 0 & 0 & 3 & 0.2 & 0.6 \\
\hline Sigmoilopsis & 1 & 0 & 0 & 0 & 0 & 0 & 0 & 0 & 0 & 0 & 0 & 0 & 0 & 0 & 0 & 0 & 0 & 1 & 0.1 & 0.2 \\
\hline
\end{tabular}

schlumbergeri

Total porcellaneous

No. species

Non fossilizing

agglutinated

agglutinant $\mathrm{spA}$

agglutinant $\mathrm{spB}$

Ammoglobigerina sp.1

Ammoglobigerina sp.2

Ammoscalaria sp.

Cystammina pauciloculata 0

Cyclammina sp.

Haplophragmoides sp.

Karreriella bradyi

Reophax sp.

Saccamina sp.

Total agglutinated

No. species

Fossilizing agglutinated

Textularia sp.

Total agglutinated

No. species

$\begin{array}{ll}6 & 1 \\ 4 & 1\end{array}$

$\begin{array}{lll}3 & 2 & 3 \\ 3 & 2 & 2\end{array}$

0
0

0

0
0

$\begin{array}{lll}0 & 0 & 0 \\ 0 & 0 & 0\end{array}$

$\begin{array}{lllrlr}0 & 0 & 0 & 20 & 0.6 & 4.2 \\ 0 & 0 & 0 & 9 & & \end{array}$

Total life foraminifera

No. species

3
4
5
0
3
3
1
0
5
0
3

1
4
1
0
0
4
1
0
0
1
1

$\begin{array}{rr}0 & 0 \\ 4 & 0 \\ 1 & 0 \\ 0 & 0 \\ 2 & 0 \\ 4 & 3 \\ 0 & (1) \\ 1 & 5 \\ 0 & 0 \\ 0 & 0 \\ 0 & 0\end{array}$

$\begin{array}{lll}0 & 0 & 0 \\ 0 & 0 & 0 \\ 0 & 4 & 2 \\ 0 & 0 & 0 \\ 0 & 0 & 0 \\ 3 & 0 & 1 \\ 1) & 0 & 0 \\ 5 & 0 & 0 \\ 0 & 0 & 0 \\ 0 & 0 & 0 \\ 0 & 0 & 0\end{array}$

$\begin{array}{rr}0 & 0 \\ 0 & 0 \\ 2 & 0 \\ 0 & 0 \\ 0 & 0 \\ 1 & 0 \\ 0 & 0 \\ 0 & (1) \\ 0 & 0 \\ 0 & 0 \\ 0 & 0 \\ & \end{array}$

0
0
1
0
0
0
0
0
0
0
0

0
0
1
0
0
0
0
0
0
0
0

$\begin{array}{lll}0 & 0 \\ 0 & 0 \\ 1 & 0 \\ 0 & 0 \\ 0 & 0 \\ 0 & 0 \\ 0 & 0 \\ 0 & 0 \\ 0 & 0 \\ 0 & 0 \\ 0 & 0\end{array}$

$\begin{array}{ll}0 & 0 \\ 0 & 0 \\ 1 & 0 \\ 0 & 0 \\ 0 & 0 \\ 0 & 0 \\ 0 \\ 0 \\ 0 \\ 0 \\ 0\end{array}$

0
0
0
0
0
0
0
0
0
0
0
0

Glomospira spp $\begin{array}{rrrrrrrr}36 & 42 & 71 & 59 & 69 & 27 & 12 & 16 \\ 17 & 14 & 20 & 15 & 14 & 8 & 6 & 3\end{array}$

Arborescent indet

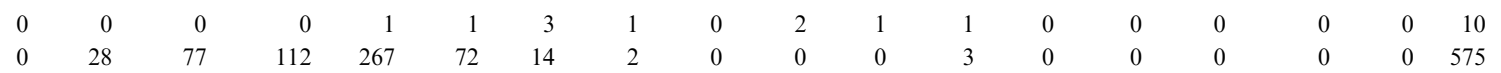


Depth


total

(d) Station F $1264 \mathrm{~m}$

Perforate

Bulimina inflata

Cibicidoides pachydermus

Cibicidoides

robertsoniu

Gavelinopsis transhucens

Glandulina ovula

Globobulimina affinis

Gyroidina umbonata

Hoeglundina elegans

Lenticulina peregrina

Melonis barleeanus

Oridorsalis umbonatus

Pullenia bulloides

Robertinoides sp.

Uvigerina mediterranea

Uvigerina peregrina

Total perforate

No. species

Porcellaneous

Quinqueloculina seminula 3

Quinqueloculina sp.

Pyrgo depressa

Total porcellaneous

No. species

Non fossilizing

agglutinated

Ammoglobigerina sp. Ammoscalaria sp.

Cyclammina sp.

Cribrostomoides

subglobosum

Karreriella bradyi

Reophax sp.

0
0
0
0
0
0

0
0
0
0
2
0

$\begin{array}{llll}1 & 0 & 0 & 0 \\ 0 & 0 & 2 & 0 \\ 3 & 0 & 0 & 0 \\ 0 & 1 & 3 & 0 \\ 0 & 0 & 1 & 0 \\ 0 & 0 & 1 & 0\end{array}$

$\begin{array}{rrrrrrrrrr}0 & 0 & 0 & 0 & 0 & 0 & 0 & 1 & 0.1 & 0.8 \\ 0 & 0 & 0 & 0 & 0 & 0 & 0 & 4 & 1.5 & 3.3 \\ 1 & 0 & 0 & 0 & 0 & 0 & 0 & 8 & 1.4 & 6.6 \\ & & & & & & & & & \\ 0 & 0 & 0 & 0 & 0 & 0 & 0 & 3 & 0.1 & 2.5 \\ 0 & 0 & 0 & 0 & 1 & 2 & 0 & 4 & 8.2 & 3.3 \\ 0 & 0 & 0 & 0 & 1 & 0 & 0 & 1 & 7.5 & 0.8 \\ 0 & 0 & 0 & 0 & 0 & 0 & 0 & 1 & 1.8 & 0.8 \\ 0 & 0 & 0 & 0 & 0 & 0 & 0 & 12 & 0.5 & 9.8 \\ 0 & 0 & 0 & 0 & 0 & 0 & 0 & 1 & 0.4 & 0.8 \\ 0 & 0 & 0 & 0 & 0 & 0 & 0 & 7 & 1.4 & 5.7 \\ 0 & 0 & 0 & 0 & 0 & 0 & 0 & 3 & 0.5 & 2.5 \\ 0 & 0 & 1 & 0 & 0 & 0 & 0 & 1 & 5.5 & 0.8 \\ 0 & 0 & 0 & 0 & 0 & 0 & 0 & 3 & 0.8 & 2.5 \\ 0 & 0 & 0 & 0 & 0 & 0 & 0 & 5 & 0.2 & 4.1 \\ 0 & 0 & 0 & 0 & 0 & 0 & 0 & 41 & 0.9 & 33.6 \\ & & & & & & & & & \\ 1 & 0 & 1 & 0 & 2 & 2 & 0 & 95 & 1.2 & 77.9 \\ 1 & 0 & 1 & 0 & 2 & 1 & 0 & 15 & & \end{array}$




\begin{tabular}{|c|c|c|c|c|c|c|c|c|c|c|c|c|c|c|c|c|c|c|c|}
\hline & \multicolumn{16}{|l|}{ Depth } & \multirow[b]{2}{*}{ Total } & \multirow{2}{*}{\multicolumn{2}{|c|}{$\mathrm{ALD}_{10} \%$ of }} \\
\hline & $0-0.25$ & $0.25-0.5$ & $0.5-0.75$ & $0.75-1$ & $1-1.5$ & $1.5-2$ & $2-2.5$ & $2.5-3$ & $3-3.5$ & $3.5-4$ & $4-5$ & $5-6$ & $6-7$ & $7-8$ & $8-9$ & $9-10$ & & & \\
\hline Saccammina sp. & 2 & 0 & 0 & 0 & 0 & 0 & (1) & 0 & 0 & 0 & 0 & 0 & 0 & 0 & 0 & 0 & 3 & 0.1 & 2.5 \\
\hline Total agglutinated & 2 & 0 & 2 & 0 & 4 & 1 & 8 & 0 & 0 & 0 & 0 & 0 & 0 & 0 & 0 & 0 & 17 & 1.5 & 13.9 \\
\hline No. species & 1 & 0 & 1 & 0 & 2 & 1 & 5 & 0 & 0 & 0 & 0 & 0 & 0 & 0 & 0 & 0 & 7 & & \\
\hline Total live foraminifera & 27 & 10 & 27 & 12 & 13 & 5 & 20 & 1 & 1 & 1 & 0 & 1 & 0 & 2 & 2 & 0 & 122 & 1.2 & 100.0 \\
\hline No. species & 9 & 6 & 8 & 5 & 6 & 3 & 10 & 1 & 1 & 1 & 0 & 1 & 0 & 2 & 1 & 0 & 25 & & \\
\hline \multirow[t]{3}{*}{ Arborescent indet } & 27 & 10 & 27 & 12 & 13 & 5 & 20 & 1 & 1 & 1 & 0 & 1 & 0 & 2 & 2 & 0 & 122 & & \\
\hline & \multicolumn{16}{|l|}{ Depth } & & & \\
\hline & $0-0.25$ & $0.25-0.5$ & $0.5-0.75$ & $0.75-1$ & $1-1.5$ & $1.5-2$ & $2-2.5$ & $2.5-3$ & $3-3.5$ & $3.5-4$ & $4-5$ & $5-6$ & $6-7$ & $7-8$ & $8-9$ & $9-10$ & Total & $\mathrm{ALD}_{10}$ & $\begin{array}{l}\% \text { of } \\
\text { total } \\
\text { fauna }\end{array}$ \\
\hline \multicolumn{20}{|l|}{$\begin{array}{l}\text { (e) Station H } 1993 \mathrm{~m} \\
\text { Perforate }\end{array}$} \\
\hline Bulimina inflata & 0 & 2 & 1 & 0 & 0 & 0 & 0 & 0 & 0 & 0 & 0 & 0 & 0 & 0 & 0 & 0 & 3 & 0.5 & 1.7 \\
\hline Cibicidoides pachydermus & 0 & 0 & 0 & 2 & 0 & 3 & 0 & 0 & 0 & 0 & 0 & 0 & 0 & 0 & 0 & 0 & 5 & 1.4 & 2.8 \\
\hline Chilostomella oolina & 0 & 0 & 0 & 0 & 0 & 0 & 0 & 0 & 0 & 0 & 0 & 0 & 1 & 0 & 0 & 0 & 1 & 6.5 & 0.6 \\
\hline Fissurina sp. & 0 & 0 & 0 & 0 & 0 & 1 & 0 & 0 & 0 & 0 & 0 & 0 & 0 & 0 & 0 & 0 & 1 & 1.8 & 0.6 \\
\hline Gavelinopsis translucens & 0 & 1 & 1 & 0 & 2 & 0 & 0 & 0 & 0 & 0 & 0 & 0 & 0 & 0 & 0 & 0 & 4 & 0.9 & 2.2 \\
\hline Globobulimina affinis & 0 & 0 & 0 & 0 & 0 & 0 & 0 & 0 & 0 & 0 & 1 & 0 & 4 & 0 & 0 & 0 & 5 & 6.1 & 2.8 \\
\hline Gyroidina umbonata & 0 & 0 & 0 & 0 & 0 & 0 & 0 & 0 & 0 & 1 & 0 & 0 & 0 & 0 & 0 & 0 & 1 & 3.8 & 0.6 \\
\hline Gyroidina orbicularis & 0 & 0 & 0 & 4 & 5 & 5 & 0 & 0 & 1 & 2 & 1 & 0 & 0 & 0 & 0 & 0 & 18 & 1.9 & 10.1 \\
\hline Hoeglundina elegans & 12 & 16 & 18 & 5 & 5 & 2 & 0 & 0 & 0 & 0 & 0 & 0 & 0 & 0 & 0 & 0 & 58 & 0.6 & 32.4 \\
\hline Lenticulina peregrina & 0 & 0 & 0 & 1 & 0 & 0 & 0 & 0 & 0 & 0 & 0 & 0 & 0 & 0 & 0 & 0 & 1 & 0.9 & 0.6 \\
\hline Pullenia sp. & 0 & 0 & 0 & 3 & 0 & 0 & 0 & 0 & 0 & 0 & 0 & 0 & 0 & 0 & 0 & 0 & 3 & 0.9 & 1.7 \\
\hline Uvigerina peregrina & 0 & 27 & 3 & 2 & 11 & 1 & 0 & 0 & 0 & (1) & 0 & 0 & 0 & 0 & 0 & 0 & 45 & 0.7 & 25.1 \\
\hline Total perforate & 12 & 46 & 23 & 17 & 23 & 12 & 0 & 0 & 1 & 4 & 2 & 0 & 5 & 0 & 0 & 0 & 145 & 1.0 & 81.0 \\
\hline No. species & 1 & 4 & 4 & 6 & 4 & 5 & 0 & 0 & 1 & 3 & 2 & 0 & 5 & 0 & 0 & 0 & 12 & & \\
\hline \multicolumn{20}{|l|}{ Porcellaneous } \\
\hline Biloculinella irregularis & 0 & 0 & 0 & 0 & 1 & 0 & 0 & 0 & 0 & 0 & 0 & 0 & 0 & 0 & 0 & 0 & 1 & 1.3 & 0.6 \\
\hline Quinqueloculina seminula & 0 & 0 & 0 & 1 & 0 & 0 & 0 & 0 & 0 & 0 & 0 & 0 & 0 & 0 & 0 & 0 & 1 & 0.9 & 0.6 \\
\hline Pyrgo depressa & 0 & 0 & 1 & 1 & 1 & 0 & 0 & 0 & 0 & 0 & 0 & 0 & 0 & 0 & 0 & 0 & 3 & 0.9 & 1.7 \\
\hline Pyrgo subsphaerica & 0 & 0 & 1 & 0 & 1 & 1 & 0 & 1 & 0 & 0 & 0 & 0 & 0 & 0 & 0 & 0 & 4 & 1.6 & 2.2 \\
\hline Scutuloris sp. & 0 & 2 & 0 & 0 & 0 & 0 & 0 & 0 & 0 & 0 & 0 & 0 & 0 & 0 & 0 & 0 & 2 & 0.4 & 1.1 \\
\hline $\begin{array}{l}\text { Sigmoilopsis } \\
\text { schlumbergeri }\end{array}$ & 1 & 2 & 0 & 0 & 0 & 0 & 0 & 0 & 0 & 0 & 0 & 0 & 0 & 0 & 0 & 0 & 3 & 0.3 & 1.7 \\
\hline
\end{tabular}


Total porcellaneous

No. species

Non fossilizing

agglutinated

Ammoscalaria sp.

Cribrostomoides

subglobosum

Cyclammina sp.

Cystammina pauciloculata

Haplophragmoides sp.

Karreriella bradyi

Reophax ampullacea

Reophax scorpiurus

Reophax sp.

Total agglutinated

No. species

Total live foraminifera

No. species

$\begin{array}{ll}2 & 0 \\ 0 & 0\end{array}$

1
2

0
0
0
0
1
0
0
0
0

$\begin{array}{llll}0 & 0 & 0 & 0 \\ 2 & 0 & 0 & 0\end{array}$

$\begin{array}{lll}0 & 0 & 2 \\ 0 & 0 & 2\end{array}$

$\begin{array}{lll}0 & 1 & 0 \\ 1 & 1 & 0\end{array}$

0

$\begin{array}{lll}0 & 0 \\ 0 & 0\end{array}$

$\begin{array}{ll}0 & 0 \\ 0 & 0\end{array}$

0

$\begin{array}{ll}0 & 0 \\ 0 & 0\end{array}$

0

$\begin{array}{lll}0 & 0 & 0\end{array}$

$\begin{array}{lll}0 & 0 & 0 \\ 0 & 0 & 0 \\ 0 & 0 & 0 \\ 0 & 0 & 0 \\ 0 & 0 & 0 \\ 0 & 0 & 0 \\ 0 & 0 & 0 \\ 0 & 0 & 0 \\ 0 & 0 & 0\end{array}$

$\begin{array}{lll}0 & 0 & 0 \\ 0 & 0 & 0\end{array}$

$\begin{array}{llllllll}0 & 0 & 0 & 0 & 0 & 3 & 0.3 & 1.7 \\ 0 & 0 & 0 & 0 & 0 & 4 & 0.9 & 2.2 \\ 0 & 0 & 0 & 0 & 0 & 2 & 0.6 & 1.1 \\ 0 & 0 & 0 & 0 & 0 & 2 & 0.6 & 1.1 \\ 0 & 0 & 0 & 0 & 0 & 2 & 0.6 & 1.1 \\ 0 & 0 & 0 & 0 & 0 & 1 & 0.4 & 0.6 \\ 0 & 0 & 0 & 0 & 0 & 3 & 0.3 & 1.7 \\ 0 & 0 & 0 & 0 & 0 & 2 & 0.1 & 1.1 \\ 0 & 0 & 0 & 0 & 0 & 1 & 0.6 & 0.6 \\ & & & & & & & \\ 0 & 0 & 0 & 0 & 0 & 20 & 1.0 & 11.2 \\ 0 & 0 & 0 & 0 & 0 & 9 & & \\ & & & & & & & \\ 0 & 5 & 0 & 0 & 0 & 179 & 1.0 & 100.0\end{array}$

Arborescent indet. 0 $\begin{array}{lll}12 & 6 & 20\end{array}$ 




Fig. 2. Abundance of the three main foraminiferal groups, and the density of foraminiferal faunas (total number of stained foraminifera per core $\left(720 \mathrm{~cm}^{3}\right)$ ) along the bathymetrical transect.

fauna (Fig. 6b) is largely limited to the upper $3 \mathrm{~cm}$ $\left(\mathrm{ALD}_{10}=1.2 \mathrm{~cm}\right)$; maximum values are found in the top first $\mathrm{cm}$. Uvigerina peregrina $(33.6 \%)$, Hoeglundina elegans (9.8\%), Cibicidoides robertsonianus (6.6\%), Melonis barleeanus (5.7\%), Quinqueloculina seminula (5.7\%), Uvigerina mediterranea $(4.1 \%)$, and Cibicidoides pachydermus $(3.3 \%)$ are the main taxa encountered at station F (Table 2d). Although the relatively low numbers of live individuals do not allow very precise observations of the vertical distribution, $Q$. seminula $\left(\mathrm{ALD}_{10}=0.3 \mathrm{~cm}\right)$ appears to be limited to the topmost $0.5 \mathrm{~cm}$, whereas $U$. peregrina $\left(\mathrm{ALD}_{10}=0.9 \mathrm{~cm}\right)$, and $H$. elegans $\left(\mathrm{ALD}_{10}=\right.$ $0.5 \mathrm{~cm}$ ) can be found slightly deeper (Fig. 6c). Just as at stations $\mathrm{B}$ and $\mathrm{A}, \mathrm{M}$. barleeanus $\left(\mathrm{ALD}_{10}=\right.$ $1.4 \mathrm{~cm}$ ) shows a subsurface maximum at about $1 \mathrm{~cm}$ depth.

At station H (water depth $1993 \mathrm{~m}$ ), the oxygen concentration is $5.85 \mathrm{ml} / \mathrm{l}$. The zero oxygen level is positioned at about $6 \mathrm{~cm}$ depth, whereas a weak nitrate + nitrite downward diffusive zone seems to be present between 2.5 and $6.5 \mathrm{~cm}$ depth (Fig. 7a). The live fauna is almost completely limited to the uppermost $2 \mathrm{~cm}$ of sediment; a maximum density (about 100 individuals $/ 50 \mathrm{~cm}^{3}$ ) is found at the $0.0-$ $0.5 \mathrm{~cm}$ level (Fig. 7b). The $\mathrm{ALD}_{10}$ of the total fauna is $1.0 \mathrm{~cm}$. Hoeglundina elegans $(32.4 \%)$,
Uvigerina peregrina (25.1\%), Gyroidina orbicularis (10.1\%), Cibicidoides pachydermus $(2.8 \%)$, and Globobulimina affinis $(2.8 \%)$ are the main taxa encountered at station $\mathrm{H}$ (Table 2d, Fig. 7c). $H$. elegans $\left(\mathrm{ALD}_{10}=0.6 \mathrm{~cm}\right)$ and $U$. peregrina $\left(\mathrm{ALD}_{10}=0.7 \mathrm{~cm}\right)$ dominate the faunas in the first half $\mathrm{cm}$. G. orbicularis $\left(\mathrm{ALD}_{10}=1.9 \mathrm{~cm}\right)$ and $C$. pachydermus $\left(\mathrm{ALD}_{10}=1.4 \mathrm{~cm}\right)$ appear mainly between 0.5 and $2 \mathrm{~cm}$ depth. Some rare individuals of G. affinis live in the deepest part of sediment, around the zero oxygen level at about $6 \mathrm{~cm}$ depth (Table 3).

\section{Discussion}

\subsection{Benthic ecosystem redox conditions}

Bottom water oxygen concentrations for our five stations vary between 4.36 and $5.85 \mathrm{ml} / 1$ (Table 1). These values agree very well with the data which have been published for the various water masses in the Bay of Biscay (Ogawa and Tauzin, 1973). The minimum values encountered at station A are typical of the Mediterranean outflow waters. Measurements of bottom water oxygenation during 10 successive sampling campaigns show only minor seasonal and interannual changes for our 
Station D

(a)
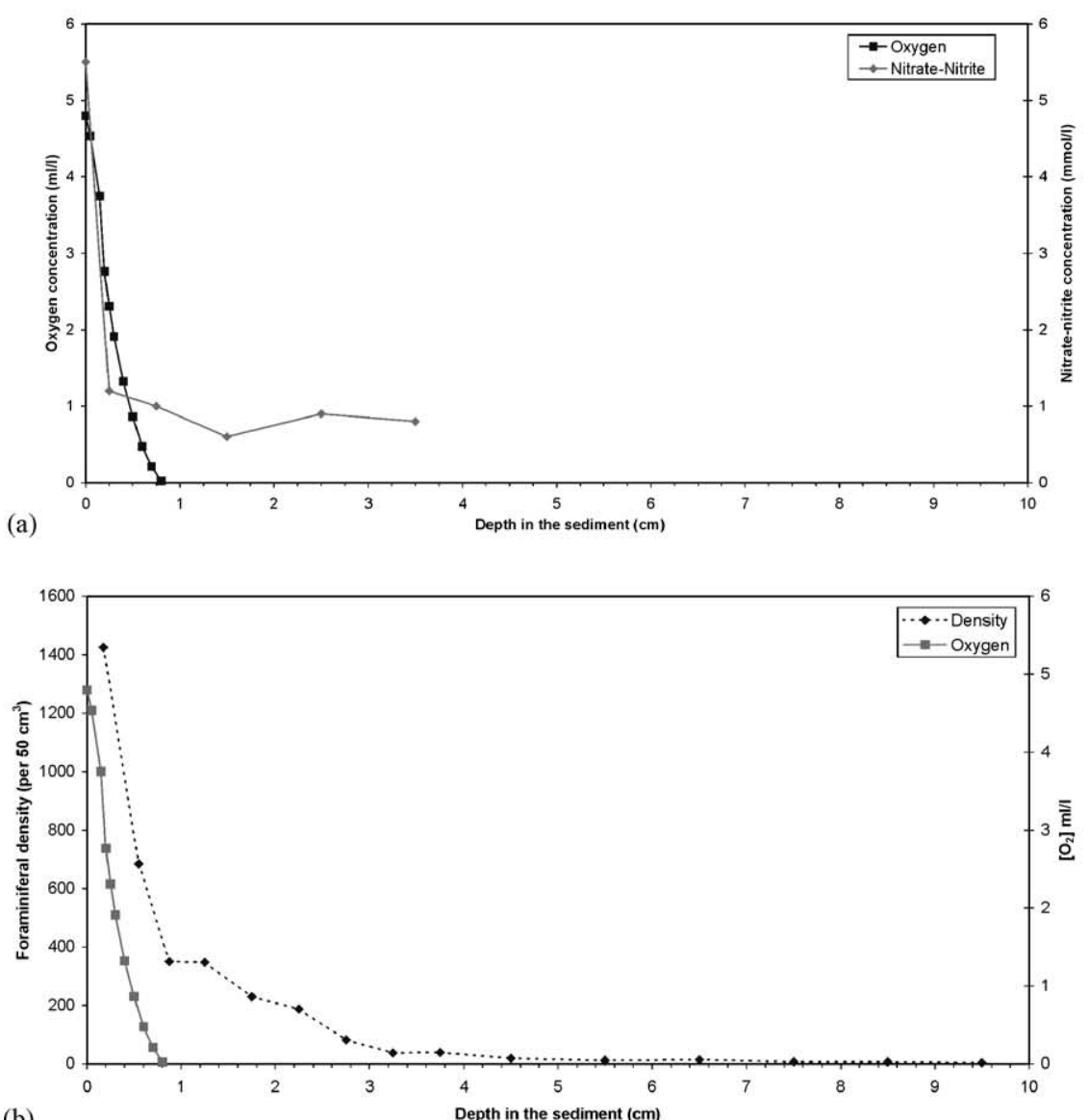

(b)

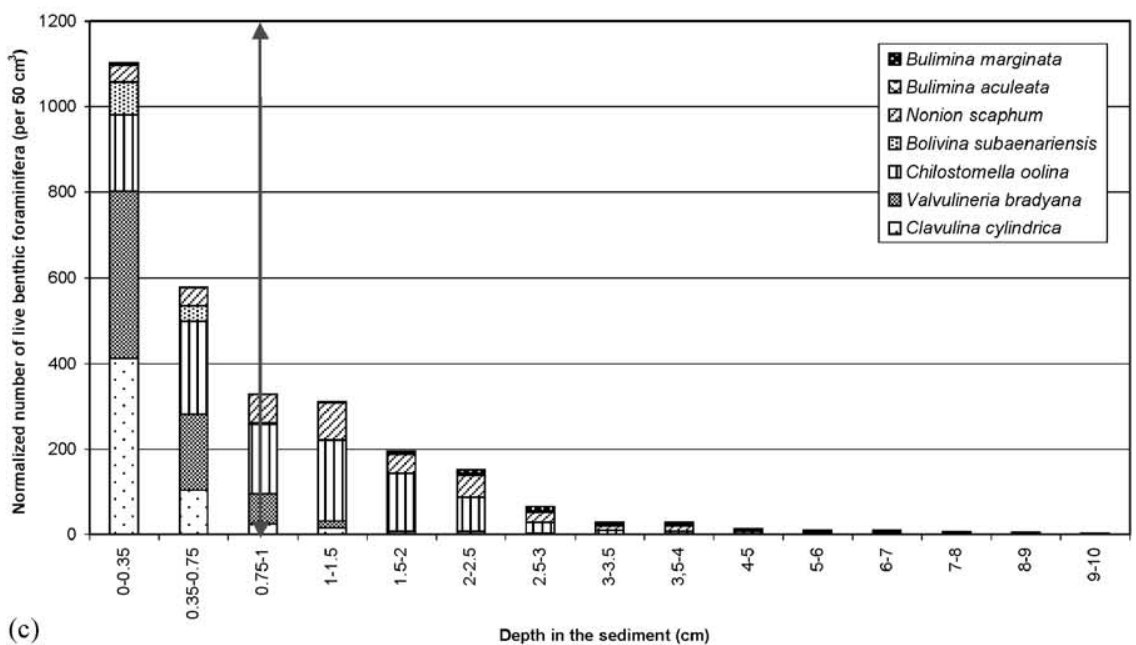

Fig. 3. Station D; (a): dissolved oxygen, and concentration of nitrate + nitrite; (b) dissolved oxygen profile and foraminiferal density (standardised for a $50 \mathrm{~cm}^{3}$ sediment volume); (c) foraminiferal distribution (number of individuals found in each level, standardised for a $50 \mathrm{~cm}^{3}$ sediment volume); double arrow represents zero oxygen boundary. 
Station B
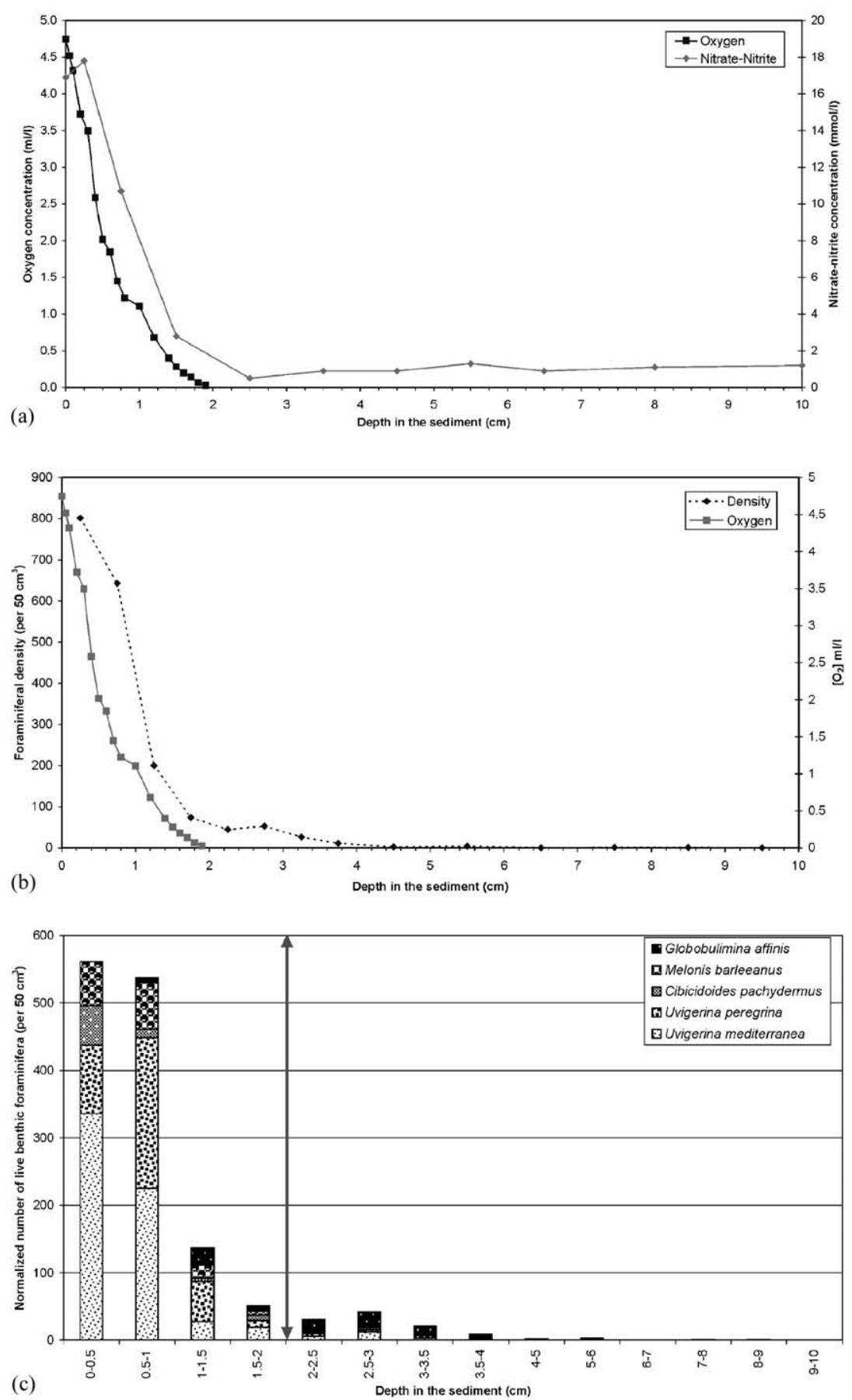

Fig. 4. Station B; (a) dissolved oxygen, and concentration of nitrate + nitrite; (b) dissolved oxygen profile and foraminiferal density (standardised for a $50 \mathrm{~cm}^{3}$ sediment volume); (c) foraminiferal distribution (number of individuals found in each level, standardised for a $50 \mathrm{~cm}^{3}$ sediment volume); double arrow represents zero oxygen boundary. 
Station A


Fig. 5. Station A; (a) dissolved oxygen, and concentration of nitrate + nitrite; (b) dissolved oxygen profile and foraminiferal density (standardised for a $50 \mathrm{~cm}^{3}$ sediment volume); (c) foraminiferal distribution (number of individuals found in each level, standardised for a $50 \mathrm{~cm}^{3}$ sediment volume); double arrow represents zero oxygen boundary. 

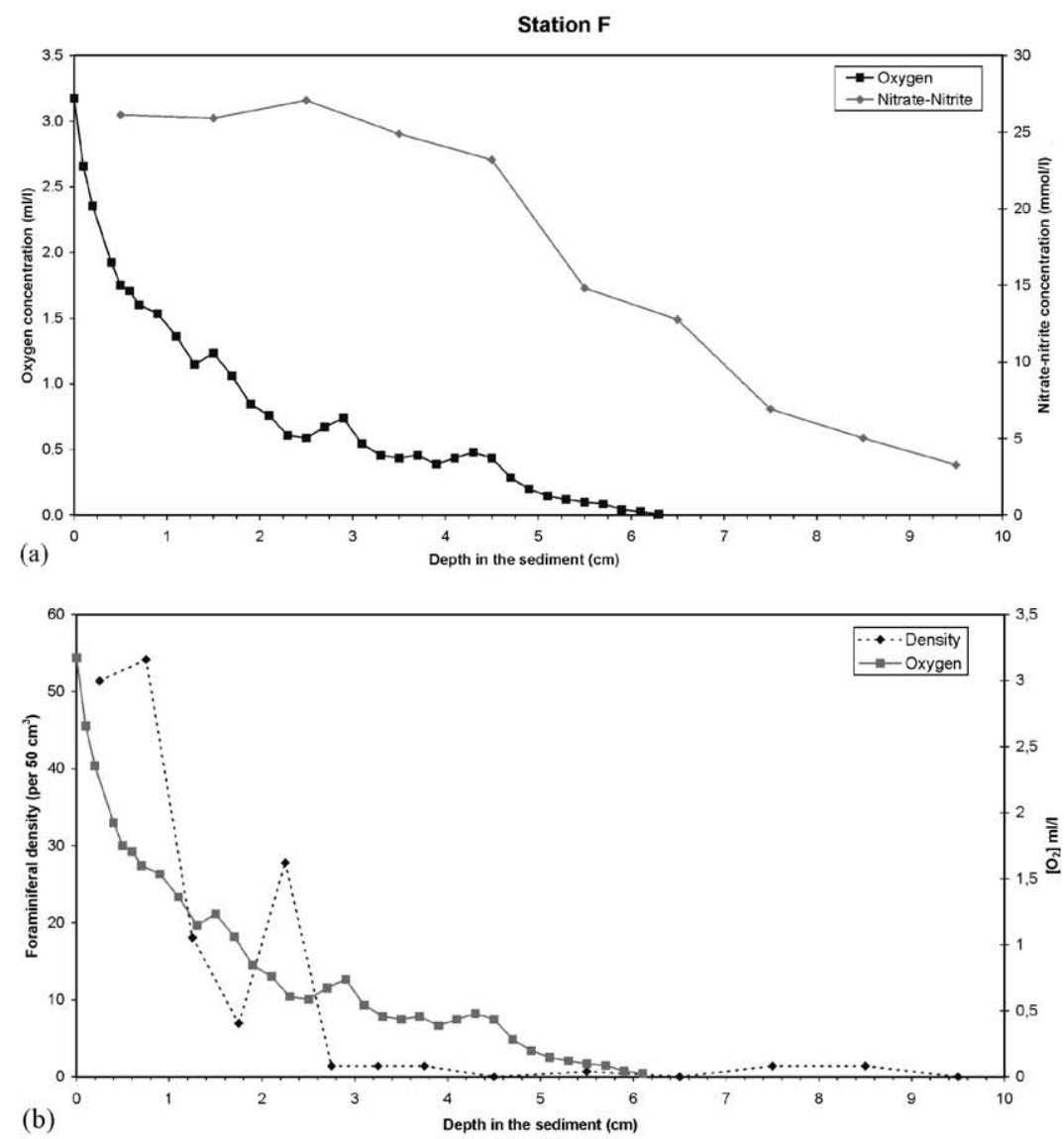

(b)

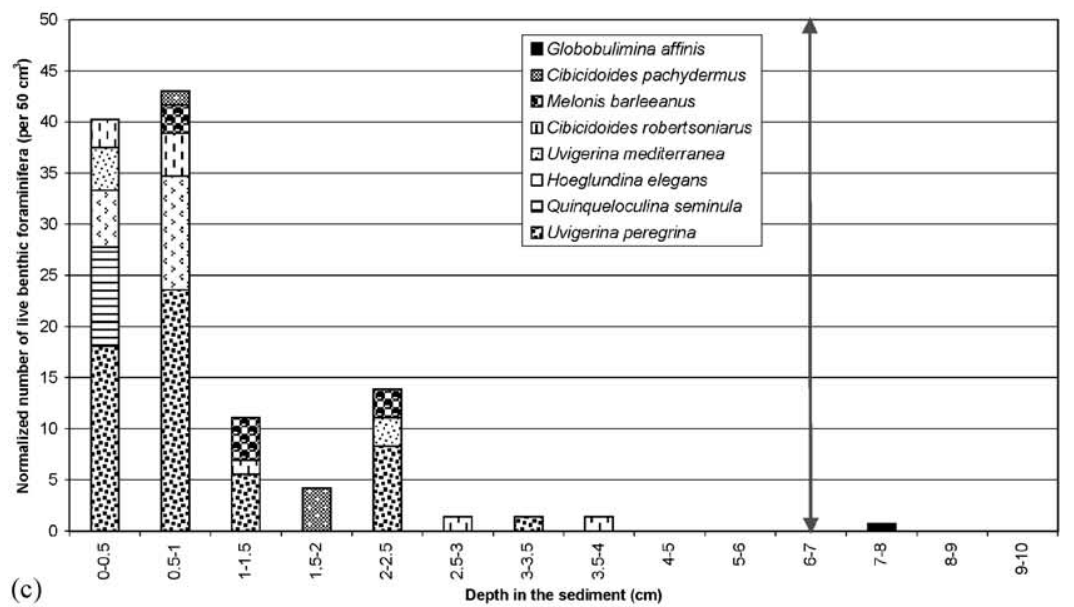

Fig. 6. Station F; (a) dissolved oxygen, and concentration of nitrate + nitrite; (b) dissolved oxygen profile and foraminiferal density (standardised for a $50 \mathrm{~cm}^{3}$ sediment volume); (c) foraminiferal distribution (number of individuals found in each level, standardised for a $50 \mathrm{~cm}^{3}$ sediment volume); double arrow represents zero oxygen boundary. 
Table 3

Average living depth $\left(\mathrm{ALD}_{10}\right)$ of foraminiferal species and (between parentheses) the number of individuals on which the calculation is based. Only occurrences of $\geqslant 5$ individuals are shown. The numbers in italic represent dominant taxa with a relative proportion $\geqslant 5 \%$ in at least one of the stations. Microhabitat patterns are summarised as shallow infaunal (SI), intermediate infaunal (II) or deep infaunal taxa (DI)

\begin{tabular}{|c|c|c|c|c|c|c|c|}
\hline \multirow[t]{2}{*}{ Species ( $>5$ specimen/core) } & \multicolumn{5}{|c|}{ Stations, $\mathrm{ALD}_{10}$ (number of specimen) } & \multirow[t]{2}{*}{ Total ALD 10} & \multirow[t]{2}{*}{ Microhabitat } \\
\hline & $\mathrm{D}$ & $\mathrm{B}$ & A & $\mathrm{F}$ & $\mathrm{H}$ & & \\
\hline Bolivina alata & $0.3(37)$ & $1.1(6)$ & & & & 0.41 & SI \\
\hline Bolivina subaenariensis & $0.3(61)$ & & & & & 0.33 & SI \\
\hline Bolivinita quadrilatera & & $0.2(7)$ & & & & 0.20 & SI \\
\hline Bulimina aculeata & $4.9(32)$ & & & & & 4.97 & DI \\
\hline Bulimina marginata & $4.2(44)$ & $1.2(19)$ & & & & 3.30 & DI \\
\hline Cancris auriculus & $0.3(11)$ & & & & & 0.35 & SI \\
\hline Cassidulina carinata & $0.7(7)$ & & & & & 0.71 & SI \\
\hline Chilostomella oolina & $1.2(600)$ & & & & & 1.20 & $I I$ \\
\hline Cibicidoides pachydermus & & $0.6(63)$ & $0.3(9)$ & & $1.4(5)$ & 0.62 & SI \\
\hline Cibicidoides robertsonianus & & & & $1.4(8)$ & & 1.40 & $I I$ \\
\hline Coryphostoma sp. & $0.2(7)$ & & & & & 0.23 & SI \\
\hline Glandulina ovula & & & $5.0(12)$ & & & 5.00 & DI \\
\hline Globobulimina affinis & $2.2(10)$ & $2.4(80)$ & $5.3(137)$ & & $6.1(5)$ & 4.24 & $D I$ \\
\hline Gyroidina altiformis & & $0.2(9)$ & & & & 0.24 & SI \\
\hline Gyroidina orbicularis & & & & & $1.9(18)$ & 1.90 & $I I$ \\
\hline Hoeglundina elegans & & & $0.8(26)$ & $0.5(12)$ & $0.6(58)$ & 0.62 & $S I$ \\
\hline Hyalinea balthica & $1.6(53)$ & $1.1(9)$ & & & & 1.52 & II \\
\hline Lenticulina peregrina & & $0.2(7)$ & & & & 0.24 & SI \\
\hline Melonis barleeanus & & $0.8(121)$ & $1.7(6)$ & $1.4(7)$ & & 0.85 & $S I / I I$ \\
\hline Nonion scaphum & $1.8(252)$ & & & & & 1.81 & $I I$ \\
\hline Nuttallides umboniferus & & $0.3(5)$ & $0.4(25)$ & & & 0.41 & $S I$ \\
\hline Pseudoeponides falsobeccarii & $3.2(25)$ & & & & & 3.20 & DI \\
\hline Rectuvigerina phlegeri & $0.5(29)$ & & & & & 0.49 & SI \\
\hline Siphogenerina sp. & & $0.6(30)$ & & & & 0.65 & SI \\
\hline Uvigerina peregrina & $0.4(38)$ & $0.8(289)$ & $1.1(85)$ & $0.9(41)$ & $0.7(44)$ & 0.79 & $S I / I I$ \\
\hline Uvigerina elongatastriata & & $1.2(60)$ & & & & 1.25 & II \\
\hline Uvigerina mediterranea & & $0.6(453)$ & $0.6(45)$ & & & 0.59 & $S I$ \\
\hline Valvulineria bradyana & $0.4(339)$ & & & & & 0.40 & $S I$ \\
\hline Quinqueloculina seminula & $0.8(45)$ & & & $0.3(7)$ & & 0.71 & SI \\
\hline Ammoglobigerina sp.1 & & & $0.6(9)$ & & & 0.64 & SI \\
\hline Ammolagena sp. & & $0.4(5)$ & & & & 0.38 & SI \\
\hline Ammoscalaria sp. & & & $0.6(10)$ & & & 0.63 & SI \\
\hline Clavulina cylindrica & $0.4(296)$ & $1(10)$ & & & & 0.38 & $S I$ \\
\hline Cribrostomoides subglobosum & & $0.5(20)$ & & & & 0.48 & SI \\
\hline Cyclammina cancellata & & $0.3(11)$ & & & & 0.26 & SI \\
\hline Cystammina pauciloculata & & & $1.1(14)$ & & & 1.12 & II \\
\hline Eggerella sp. & $0.5(10)$ & $0.6(6)$ & & & & 0.52 & SI \\
\hline Haplophragmoides sp. & $0.4(8)$ & & $1.7(6)$ & & & 0.94 & II \\
\hline Karreriella bradyi & & & $0.5(7)$ & & & 0.52 & SI \\
\hline Nouria polymorphinoides & $0.4(16)$ & & & & & 0.36 & SI \\
\hline Reophax sp. & $0.3(6)$ & $0.4(52)$ & & & & 0.40 & SI \\
\hline Saccammina sp. & & & $0.6(6)$ & & & 0.58 & SI \\
\hline Bigenerina nodosaria & & $0.4(9)$ & & & & 0.36 & SI \\
\hline Pseudoclavulina crustata & & $0.9(10)$ & & & & 0.93 & II \\
\hline Siphotextularia affinis & & $0.5(9)$ & & & & 0.51 & SI \\
\hline
\end{tabular}



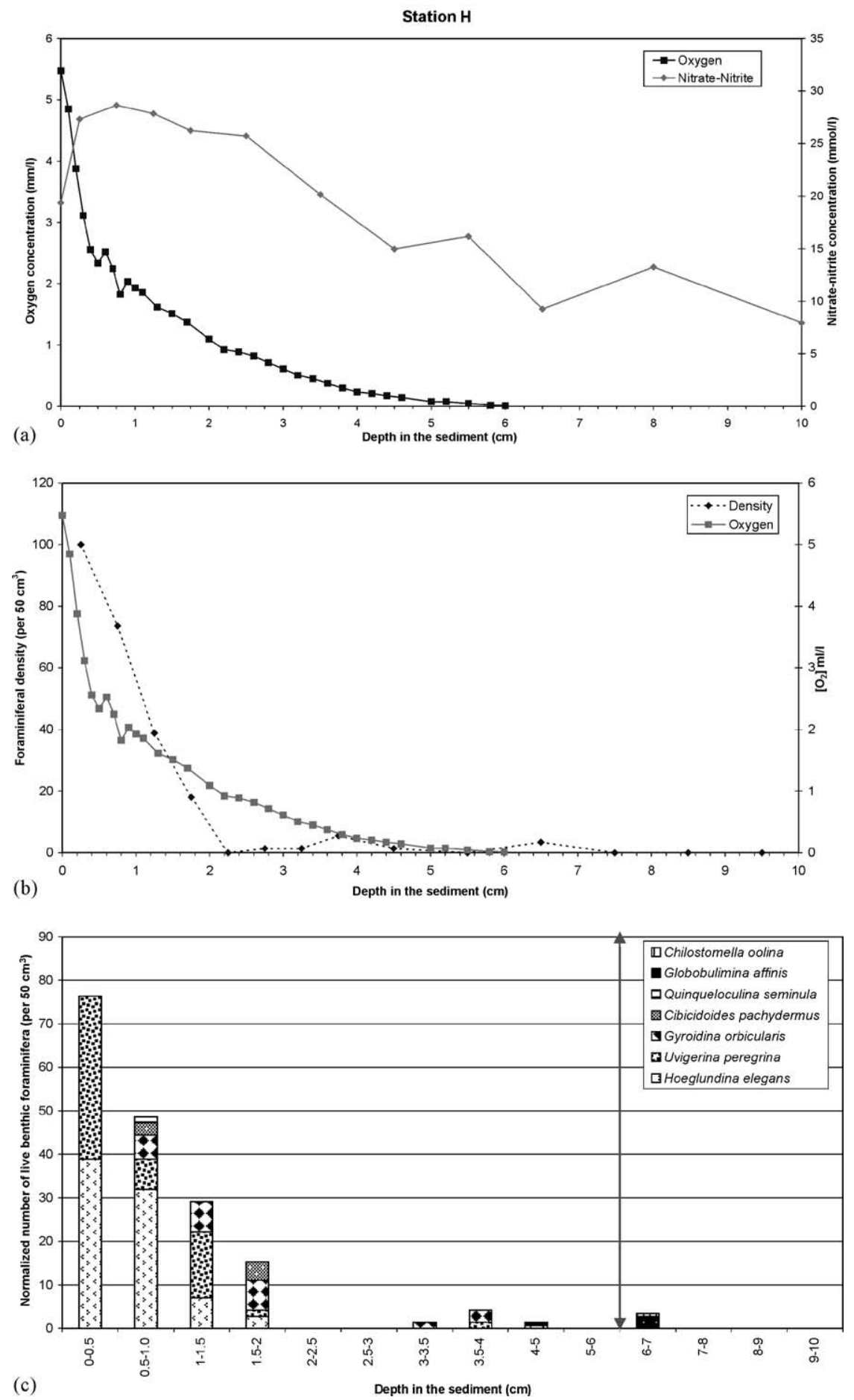

Fig. 7. Station H; (a) dissolved oxygen, and concentration of nitrate + nitrite; (b) dissolved oxygen profile and foraminiferal density (standardised for a $50 \mathrm{~cm}^{3}$ sediment volume); (c): foraminiferal distribution (number of individuals found in each level, standardised for a $50 \mathrm{~cm}^{3}$ sediment volume); double arrow represents zero oxygen boundary. 
five stations (Anschutz et al., 1999; Hyacinthe et al., 2001), suggesting that the oxygen concentration of the bottom waters is only very weakly influenced by the variability of the flux of organic matter to the ocean floor. The penetration of free oxygen into the sediment varies from only $8 \mathrm{~mm}$ at the shallow station D to more than $6 \mathrm{~cm}$ at the deep stations $\mathrm{F}$ and $\mathrm{H}$ (Table 1). These values strongly suggest that the oxygen gradient in the interstitial waters is only weakly dependent on bottom water oxygenation, but is strongly influenced by the rate of oxygen consumption within the sediment. This rate depends on the oxic degradation of organic matter and oxidation of upward diffusing reduced components. But the oxygen penetration depth is relatively stable through the year (Anschutz et al., 1999; Hyacinthe et al., 2001). Our flux calculations, based on primary production and water depth, suggest a labile organic carbon flux to the ocean floor that is about 14 times higher in station D (where oxygen penetration is minimal) than at station $\mathrm{H}$ (where oxygen penetration is maximal).

As soon as all free oxygen has been consumed for the degradation of reactive organic matter, other oxidants are used to continue the remineralisation of labile organic compounds (Froelich et al., 1979; Fenchel and Finlay, 1995). A first step of anaerobic degradation is the reduction of nitrate and nitrite in dinitrogen. This redox reaction takes place mostly below the zero oxygen level, and causes an upward diffusion of newly formed ammonia from the anoxic to oxic layers. In hypoxic sediments, ammonia is oxidised by nitrifying bacteria in nitrate and nitrite, which results in a downward diffusion of nitrate (and nitrite) from oxic to anoxic layers. At the shallowest station $\mathrm{D}$, where the organic flux is maximal, the zone of nitrate reduction is probably situated in the uppermost $\mathrm{cm}$ of the sediment (Fig. 3a). Towards the deeper, more oligotrophic stations, the zone of downward diffusion of nitrate + nitrite gradually deepens, from $0.25-2.25 \mathrm{~cm}$ at station $\mathrm{B}$ to $0.75-3.5 \mathrm{~cm}$ at station $\mathrm{A}$, to about $2.5-7.5 \mathrm{~cm}$ at stations $F$ and $H$. Sulphate reduction may be another important mechanism involved in the anaerobic degradation of organic matter; this phenomenon typical of eutrophic ecosystems was observed only in station D. Important bacterial consortia are supposed to induce these anaerobic redox reactions, which ultimately result in almost complete degradation of both labile and refractory organic matter (Hargrave, 1970; Carney, 1989). The bacterial consortia may constitute an important additional source of labile organic matter below the oxic zone, and could trigger an important recycling of organic carbon under anaerobic conditions (Fenchel and Finlay, 1995).

\subsection{Faunal characteristics}

It is generally accepted that organic mater influences the composition of the foraminiferal fauna both qualitatively and quantitatively (Thiel, 1983; Berger and Diester Haas, 1988; Altenbach and Sarnthein, 1989; Herguera and Berger, 1991; Altenbach, 1988; Gooday, 1993; Jorissen et al., 1998). In our study area, the faunas of outer shelf station D contain about 15 times more stained foraminifera than those of the deeper stations $F$ and $\mathrm{H}$ (Fig. 2). This difference is probably induced by the increase of the vertical labile organic matter flux to the ocean floor towards shallow water. Also the number of species varies between the stations; by far the highest number (49) of taxa is found at station $\mathrm{B}$. This elevated number can probably be explained by the fact that the fauna contains both outer shelf and upper slope elements. The deepest stations $\mathrm{F}$ and $\mathrm{H}$ have by far the lowest number of taxa, but this is probably at least partially due to the fact that the samples contain about 15 times fewer individuals here.

At all stations, the live faunas are strongly concentrated in the first $\mathrm{cm}$ of the sediment, suggesting a dependence on the flux of labile, easily consumable organic matter. Despite the strong faunal concentration in the first $\mathrm{cm}$, the $\mathrm{ALD}_{10}$ of the total faunas (Fig. 8a and b) is not uniform: between 0.8 and $1.2 \mathrm{~cm}$ for the relatively shallow stations D and B, as well as for the much deeper stations $\mathrm{F}$ and $\mathrm{H}$, but about $2.4 \mathrm{~cm}$ for station A $(1012 \mathrm{~m})$. The deepening of the $\mathrm{ALD}_{10}$ for the total fauna at station $\mathrm{A}$ is mainly the result of an important increase of the $\mathrm{ALD}_{10}$ of the infaunal species G. affinis and C. oolina, which are dominant faunal elements here (with a cumulative 

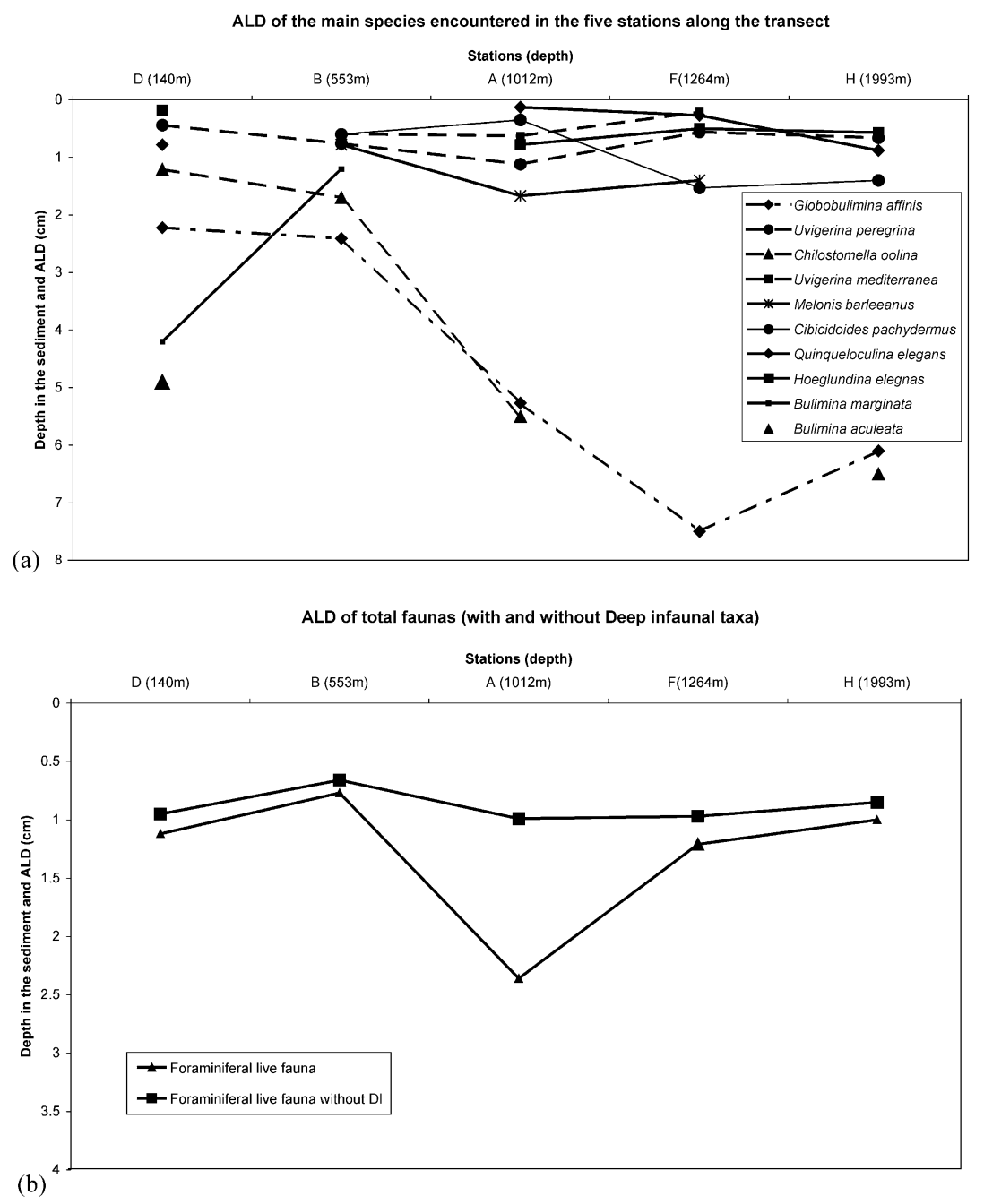

Fig. 8. (a) $\mathrm{ALD}_{10}$ of the main taxa along the bathymetric transect; (b) $\mathrm{ALD}_{10}$ of the total faunas (with and without deep infaunal taxa (DI)).

percentage of 30\%) (Fig. 8a). Although their living depth further deepens at the deeper stations $\mathrm{F}$ and $\mathrm{H}$, their relative proportion (and thus also their influence on the total faunal $\mathrm{ALD}_{10}$ ) decreases strongly there. Thus, the very shallow $\mathrm{ALD}_{10}$ of the latter 2 stations is caused mainly by the scarcity of deep infaunal elements. When we consider the $\mathrm{ALD}_{10}$ of the total faunas without these two potentially deep infaunal species (Fig. 8b), the result is a very stable $A_{L D}$ of all superficially living taxa (between 0.5 and $0.8 \mathrm{~cm}$ ).
The shallower station D contains about 2000 individuals that are largely restricted to shallow and intermediate infaunal positions. Apparently this fauna reflects a high labile organic flux to the ocean floor. Valvulineria bradyana and Clavulina cylindrica dominate the first half $\mathrm{cm}$. They can be considered as two shallow infaunal species, tolerant of low oxygen values, which respond to a high food level at the sediment-water interface. The first species has been described in low oxygen sediments from the centre of the Adriatic Sea mud belt 
(Jorissen, 1987, 1988; Van der Zwaan and Jorissen, 1991). The dominant species at this station, Chilostomella oolina, has been described as an intermediate or deep infaunal species well adapted to suboxic conditions (Corliss, 1985; Van der Zwaan and Jorissen, 1991; Sen Gupta and Machain-Castillo, 1993; Bernhard and Sen Gupta, 1999). It settles together with Nonion scaphum in strongly suboxic and anoxic sediments down to 3$\mathrm{cm}$ depth. In these stressed environments, these two species appear to have a competitive advantage over more superficially living taxa. They may proliferate because of the input of large quantities of organic matter into the deeper sediment layers, remineralised by anaerobic pathways, and the near-absence of less resistant competing taxa (Rathburn and Corliss, 1994). Next, an association consisting of Bulimina aculeata, Bulimina marginata, and Pseudoeponides falsobecarii is found deep in the sediment, under completely anoxic conditions. Several authors (e.g. Lutze and Coulbourn, 1984; Jorissen, 1987; Hermelin and Shimmield, 1990; Verhallen, 1987; Bernhard and Alve, 1996) have described Bulimina species as typical elements in extremely eutrophic and dysoxic settings. In view of their consistent distribution with a surface as well as a deep maximum (Jorissen, 1999, Figs. 10.6d and 10.7f), and the systematic association with sea urchins in our material, we think that the deep occurrences of this association can be explained by their colonisation of macrofaunal burrows. These environments could be attractive because of the strongly increased bacterial activity in the burrow walls (e.g. Fenchel and Jørgensen, 1977).

A major faunal change takes place between stations D (140 m) and B (553 m) (Fig. 9). The latter station is strongly dominated by Uvigerinids (U. mediterranea and U. peregrina). These shallow infaunal species have been described in a wide variety of eutrophic settings, needing an exported labile organic flux of at least $2.5 \mathrm{~g} \mathrm{C} / \mathrm{m}^{2} / \mathrm{yr}$ (e.g. Lutze and Coulbourne, 1984; Corliss, 1985; Corliss and Emerson, 1990; Corliss, 1991; De Rijk et al., 2000; Morigi et al., in press). They can be considered as feeding on labile organic matter in rather well oxygenated shallow infaunal microhabitats. It is important to notice that $U$. peregrina

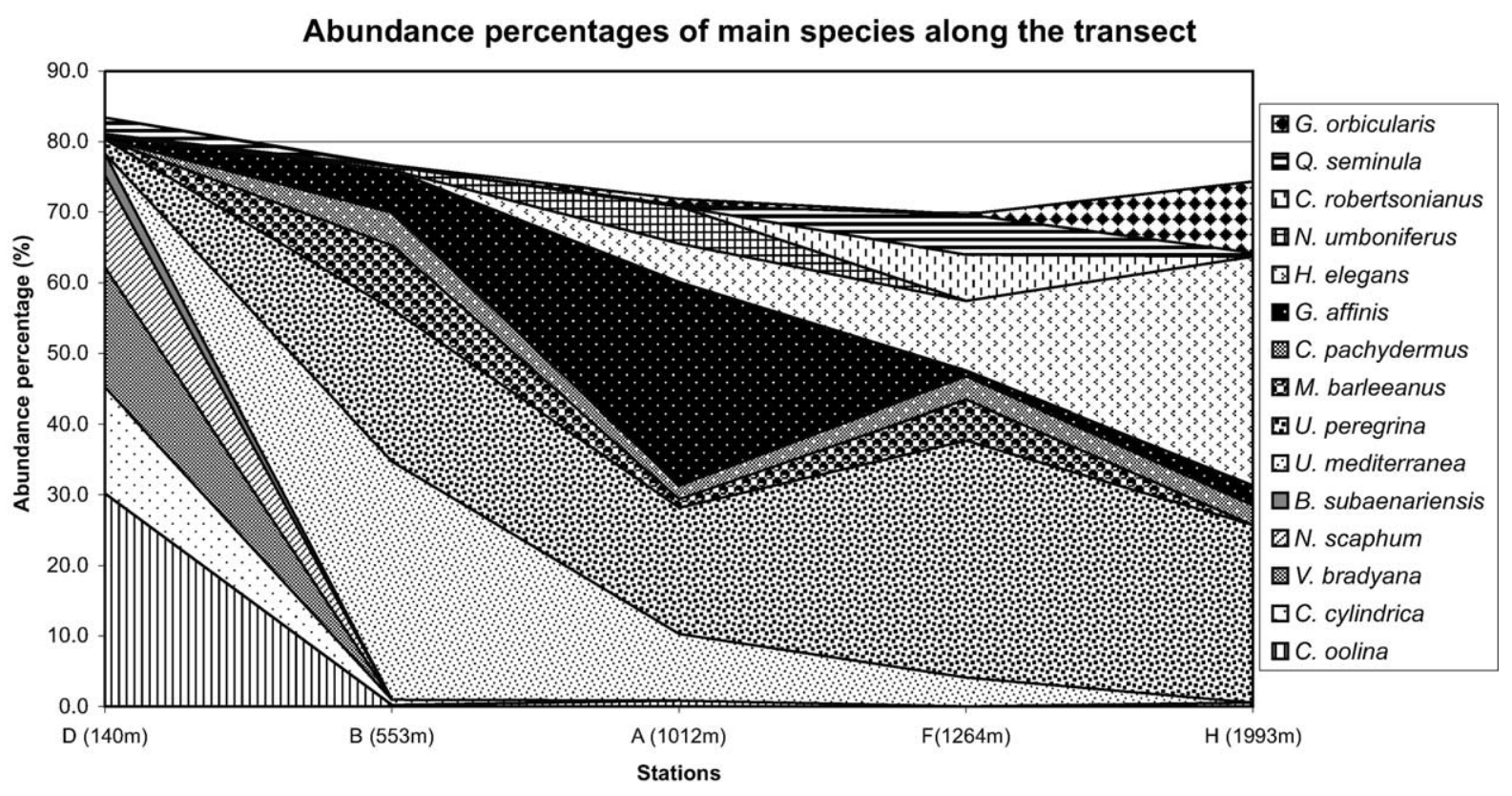

Fig. 9. Composition of the benthic live foraminiferal faunas (in $\%$ of the total fauna). 
is consistently found deeper than U. mediterranea. This could be caused by a slightly higher tolerance for dysoxic conditions. A study of the seasonal variation at station B, however (Fontanier et al., in preparation), shows rather important changes of the microhabitat depth of both taxa through the year, in a context where the oxygen profiles show a very limited variability. Both taxa show maximum microhabitat depths in the richest samples (taken in the most productive periods), which strongly suggests a dependency of the living depth on the quality of the organic matter, high quality organic matter being more abundant in deeper sediment layers during the most eutrophic periods. The deeper microhabitat of $U$. peregrina would then show a slightly higher tolerance for low quality organic matter. A consequence of our observations is that the $\delta^{13} \mathrm{C}$ of $U$. peregrina can hardly be considered as typical of bottom waters.

Deeper in the sediment, the infaunal niches are occupied by $M$. barleeanus and (still deeper) $G$. affinis. This combination of species has been described in numerous mesotrophic-eutrophic oceanic ecosystems (e.g. Harloff and Mackensen, 1997). Jorissen et al. (1995) observe that $M$. barleeanus occurs systematically in the lower part of the oxic zone, where nitrate production (by nitrifying bacteria) occurs, whereas G. affinis is consequently found in the upper part of the totally anoxic zone, where nitrate reduction occurs. Our results confirm this pattern. They suggest that both species are dependent on aerobic and anaerobic bacterial stocks degrading more or less refractory organic matter. These bacterial stocks should be concentrated directly below the major redox fronts, where maximum foraminiferal abundances have been described (e.g. Mullins et al., 1985; Linke and Lutze, 1993; Thomsen and Altenbach, 1993; our data). Both species would either prey directly on the bacterial stocks (Lee, 1979; Thomsen and Altenbach, 1993; Kitazato, 1994), or feed themselves with the break-off products. Our data confirm these ideas. We assume that most of the labile components will be consumed in the well-oxygenated topmost $\mathrm{cm}$ of the sediment, and will not arrive at the depth where $M$. barleeanus and G. affinis live. In the much more eutrophic station $\mathrm{D}$, on the other hand, the near- absence of both species may be explained by the availability of important amounts of labile organic matter in the sub-surface dysoxic and anoxic levels, which are colonised by $C$. oolina and $N$. scaphum, species that appear to be less able to subsist on low quality organic matter. The total dominance of $C$. oolina in the levels just below Mediterranean sapropel S1 (Jorissen et al., in preparation), which were deposited in a strongly dysoxic setting with an important labile organic matter influx, would be another argument in favour of this interpretation.

Dominant taxa found at station A are essentially the same as those found in station B, showing that the change from North Atlantic Central Waters to MW has no visible impact on the benthic faunas. The almost perfect separation between the group of superficial infaunal taxa (dominated by Nuttallides umboniferus, U. peregrina and $U$. mediterranea) from a deep infaunal assemblages (dominated by Globobulimina affinis) is striking. The appearance of more oligotrophic shallow infaunal taxa such as $H$. elegans, $N$. umboniferus and C. pachydermus (compare Corliss, 1985; Corliss and Emerson, 1990; Corliss, 1991), shows the influence of the gradually decreasing organic flux towards deeper areas. Also rather oligotrophic arborescent agglutinated taxa (Jones and Charnock, 1985), which have not been included in our counting results, are very abundant at this station (575 fragments). The high quantities of $G$. affinis in the anoxic sediments from 2 to $8 \mathrm{~cm}$ depth can be explained in several ways. They could be (1) dead, but still staining, partially decomposed individuals, (2) live, but inactive animals with a lowered metabolism, or (3) active, facultative aerobic individuals, which dwell on an important stock of food made available by bacterial activity. The source of the originally (before bacterial conversion/partial break-off) refractory organic matter could be a former refractory organic matter input by slope failure processes.

The poor faunas (in terms of faunal density) of stations F (1264 m) and H (1993 m) show faunal changes characterising the further decrease of the trophic level. U. mediterranea and G. affinis show a strong relative frequency decrease, and $U$. pere- 
grina (station F) and H. elegans become dominant. Furthermore, the faunas become largely restricted to the well-oxygenated top $2 \mathrm{~cm}$ of the sediment. The slightly deeper zone of nitrate reduction is almost devoid of benthic foraminifera. In the top of the anoxic zone, only very few individuals of $G$. affinis are found. This faunal evolution translates the trend towards oligotrophic ecosystems, where metabolisable organic matter is limited to the uppermost sediment layers. Apparently bacterial conversion of refractory organic matter in deeper, dysoxic/anoxic sediment layers is a minor process here.

\subsection{Benthic foraminiferal microhabitats}

The dependence of the benthic foraminiferal microhabitat on the availability of food and the oxygenation of the benthic ecosystem has been schematised in a conceptual model by Jorissen et al. (1995), as shown in Fig. 10. The so-called TROX-model explains that in very oligotrophic environments, shallow infaunal species, which are adapted to a low trophic level, will thrive close to the sediment-water interface in a well oxygenated setting. The scarcity of organic matter introduced into the sediment (because of weak bioturbation and almost complete consumption of organic compounds in the first $\mathrm{mms}$ of the sediment)

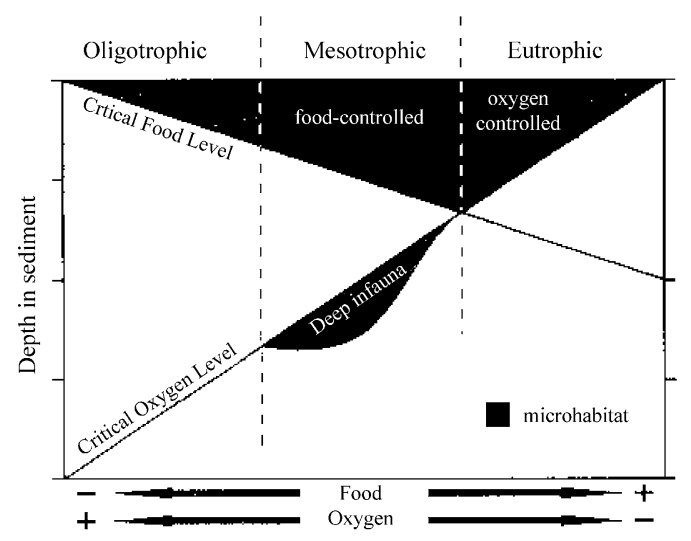

Fig. 10. TROX-model (Trophic condition and Oxygen concentration) explaining the vertical distribution of foraminifera in the top $\mathrm{cm}$ of the sediment (after Jorissen et al., 1995); see text for full explanation. prevents colonisation of the deeper sediment layers by infaunal taxa. But also in much more eutrophic conditions, where the principal redox front is positioned close to the sediment surface, shallow infaunal taxa are limited to the first $\mathrm{mm}$ of the sediment; in this case, they have only a limited tolerance for low oxygen conditions. According to the TROX-model, faunal penetration will be maximal in mesotrophic settings, where oxygen penetration is relatively deep, and more or less labile food particles are introduced at depth in the sediment by bioturbating macrofauna. In such mesotrophic environments, grazing on anaerobic bacterial stocks (or their break-up products) has been proposed as an explanation for the presence of rather deep-living intermediate and deep infaunal species. It is evident that the foraminiferal taxa have a dynamic behaviour, allowing them to adapt rapidly to changing conditions. Inherent to the TROX-model is the notion that the control of the foraminiferal microhabitat is dual. Whereas oxygenation is considered as the main controlling parameter in eutrophic ecosystems, food availability is supposed to control the vertical faunal distribution in more oligotrophic environments.

Our data fully confirm the main distributional trends predicted by the TROX-model. At stations $\mathrm{D}$ and $\mathrm{B}$, which represent the eutrophic extreme in our study, the faunas are concentrated in the first $2 \mathrm{~cm}$. In the "mesotrophic" station A, faunal penetration is deepest, and an important population of deep infaunal $G$. affinis is found at considerable depth in the sediment. Stations F an $\mathrm{H}$, finally, represent the most oligotrophic situation in our study, and faunas are once again limited to the first centimetres of the sediment.

Despite the good fit to our data, it is evident that the TROX-model oversimplifies the foraminiferal response to the controlling environmental parameters for a number of reasons:

(1) The TROX-model does not insist enough on the dependency of pore water oxygenation on the organic flux. Since the supply of metabolisable organic matter controls the oxygen consumption in the sediment and the localisation of the successive redox fronts, it is evident 
that the organic flux is the main parameter controlling the foraminiferal distribution in the sediment.

(2) Recent studies (e.g. Alve and Bernhard, 1995; Fenchel and Finlay, 1995; Moodley et al., 1997; Jannink et al., 1998) show that anoxic conditions do not have a direct lethal effect for the majority of species. If severe dysoxia in bottom and pore waters cause the disappearance of certain taxa, this is possibly so, because reproduction is inhibited in such environments. Therefore, benthic ecosystem oxygenation appears to be a less important factor than was suggested by Jorissen et al. (1995), and in consequence, the quantity and quality of food particles appear to be by far the most important parameters.

(3) The TROX-model depicts trends for the total fauna, but individual taxa may show important differences in their tolerance levels with respect to the main controlling parameters.
(4) It is evident that competition between taxa for high quality food particles is an important factor causing microhabitat differences (Van der Zwaan et al., 1999). The deep microhabitat of several infaunal taxa may be caused by their low competitiveness in the more attractive sediment surface niches (which are much richer in easily metabolisable organic matter).

(5) Benthic ecosystems may know important seasonal or interannual variability. The foraminiferal faunas will respond to rapid changes in the organic flux or bottom water oxygenation by a shift of their microhabitat structure (Barmawidjaja et al., 1992; Ohga and Kitazato, 1997).

In Fig. 11, we show the microhabitat occupation at a species level in the range of meso-oligotrophic environments represented by our stations. Labile, particulate organic matter will be concentrated at the sediment surface. In extremely oligotrophic

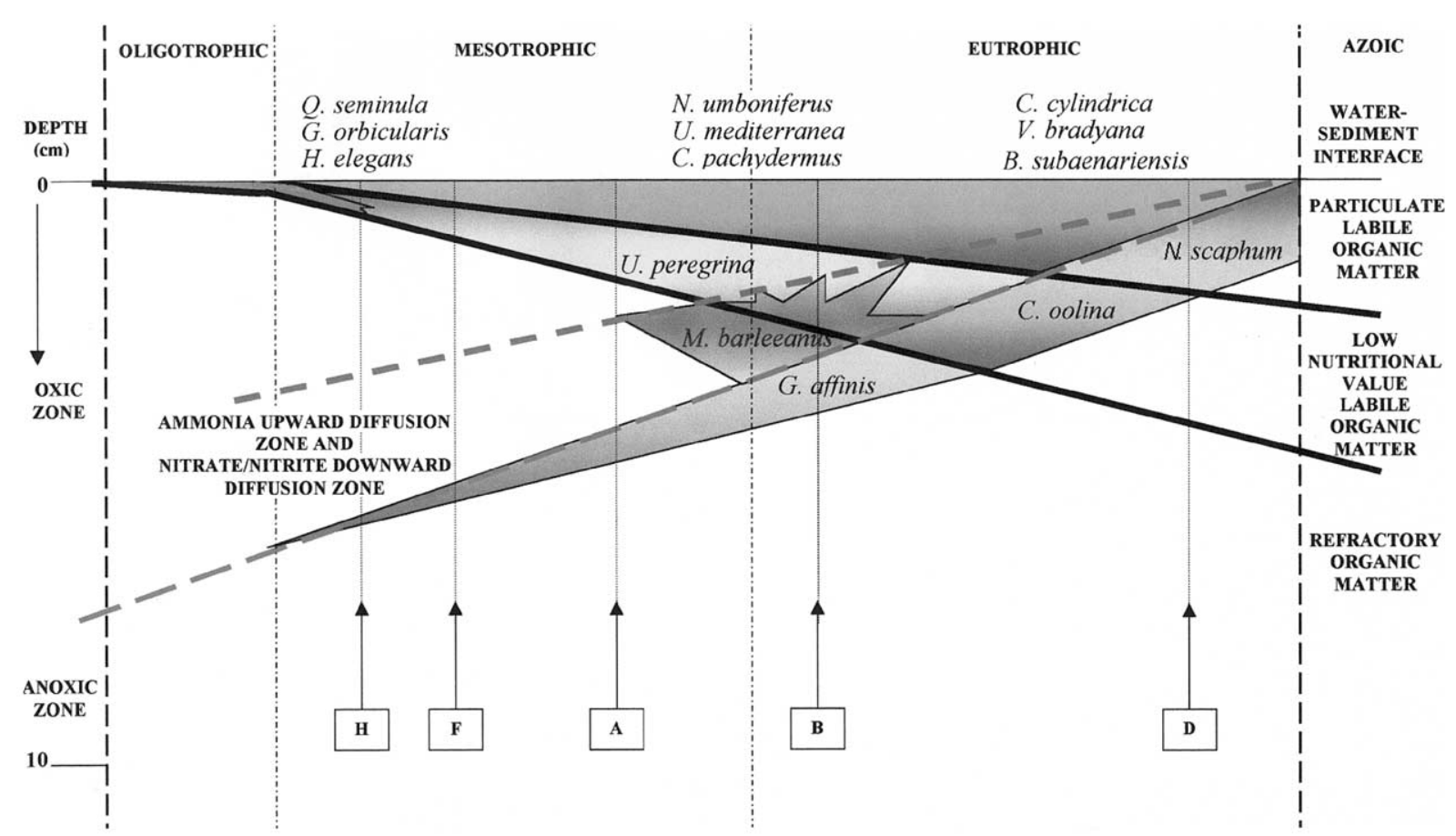

Fig. 11. Microhabitat distribution and specific foraminiferal composition along the bathymetric transect in the Bay of Biscay; the approximate position of our five stations are indicated; see text for full explanation. 
ecosystems such easily metabolisable material will be very scarce, or even be absent, except for rare phytodetritus deposit events (Gooday, 1993). Towards slightly less oligotrophic sites (our stations $\mathrm{H}$ and $\mathrm{F}$ ), part of this material will be introduced in the top $\mathrm{mm}$ of the sediment. The result is the creation of a niche inhabited by very competitive, shallow infaunal taxa. In rather oligotrophic settings such faunas are dominated by $H$. elegans, $Q$. seminula, and C. pachydermus, in more mesotrophic settings (stations A, B) by $U$. mediterranea, N. umboniferus and G. orbicularis, and in more eutrophic environments (station D) by $C$. cylindrica, $V$. bradyana and $B$. subaenariensis (Fig. 11). All these taxa seem to combine a preference for high quality resources (or a low tolerance for low quality food), a high competitiveness and perhaps a limited tolerance for anoxic conditions. When in eutrophic ecosystems, labile, easily metabolisable matter is introduced into anoxic parts of the sediment column (because of a shallow zero oxygen level), other, more lowoxygen resistant species, such as $C$. oolina and $N$. scaphum, will take over this superficial niche. Immediately below this surface zone with easily metabolisable organic matter, an environment is found where organic matter is less reactive (for instance in faecal pellets). U. peregrina seems to be one of the most tolerant species for this lower quality organic matter. In oligotrophic ecosystems, at the basal part of this zone, reactive organic matter becomes very scarce, or even absent within the sediment, and, consequently, benthic foraminifera are no longer found. Still deeper, concentrations of reactive organic matter will be associated with bacterial activity, and will logically be positioned around important redox boundaries (Lee, 1979; Mullins et al., 1985; Linke and Lutze, 1993; Kitazato, 1994; Jorissen, 1999). One of the first of these fronts may be that of nitrifying bacteria. Between this front and the zero oxygen level, M. barleeanus seems to be the best-adapted taxon. The main locus of bacterial activity appears to be concentrated in the topmost part of the anoxic layer. Here, abundant benthic foraminiferal faunas dominated by G. affinis may be present. In the more oligotrophic environments (stations $\mathrm{H}$ and F), the burial of organic compounds becomes insignificant and can no longer sustain important anaerobic bacterial stocks and associated deep infaunal foraminifera. When reading Fig. 11, one should, of course, realise that the limits between the various types of resources are not sharp, and consequently, large overlaps between the various taxa occur. Furthermore, ecosystems are always dynamic. The trophic level may show important short-term fluctuations, and the benthic faunas will respond accordingly. In general, more opportunistic taxa will profit from such ecosystem instability.

\section{Conclusions}

1. The dissolved oxygen concentration of the bottom waters is not seriously influenced by the exported organic flux. The interstitial waters, on the contrary, show a clear linkage: at the shallowest site (D), high and relatively constant organic matter inputs result in a redox front close to the sediment-water interface. At the deeper sites, the zero oxygen level is found much deeper in the sediment, as a direct result of the decreasing organic flux.

2. The flux of organic matter to the ocean floor is the main parameter that controls the density and the composition of benthic foraminiferal faunas along the bathymetrical transect.

3. The oxygenation of the pore waters does not have a direct control on the vertical distribution of most foraminiferal taxa. Most species are able to adapt to severe dysoxia or even anoxia, provided that sufficient high quality food particles are available.

4. The TROX-model (Jorissen et al., 1995) is confirmed by the present data. Faunal penetration is indeed maximal in mesotrophic conditions, where the advantageous conditions of high-oxygen concentration and ample high quantity food availability may be found relatively deep in the sediment. However, the variability of the total faunal $\mathrm{ALD}_{10}$ (Average Living Depth) is mainly determined by deep infaunal taxa. Most shallow infaunal taxa show only a minor variability in their microhabitats between the various stations. 
5. Certain taxa, such as $N$. scaphum and C. oolina appear to be typical for settings where labile organic matter is introduced into strongly dysoxic or anoxic environments. In case of an organic matter of lower quality, they are replaced by $M$. barleeanus and G. affinis.

6. Rather consistently, $U$. peregrina is found slightly below the most superficially living taxa, confirming that it is incorrect to consider the stable isotopic composition of its shell as representative for the bottom waters.

\section{Acknowledgements}

We thank the crews of Côte de la Manche for good collaboration during the OXYBENT cruises. We are grateful to two anonymous reviewers for their helpful comments on the original manuscript. This work has been carried out within the framework of the French national program PROOF.

\section{Appendix A}

Differentiating between Uvigerina peregrina and Uvigerina mediterranea may be difficult sometimes. This is especially the case when $U$. peregrina types lose the spinose aspect of their ornementation. A good overview of various $U$. peregrina morphotypes is given in Van der Zwaan et al. (1986), dealing with Uvigerina taxonomy.

In general, differences between the 2 species are as follows:

(1) U. mediterranea has a rather bulky test, with a low length/width ratio, the opposite is true for
$U$. peregrina. Adult $U$. mediterranea are much bigger than adult $U$. peregrina.

(2) $U$. mediterranea has a rather large first chamber, much bigger than $U$. peregrina. Exception: microspheres of $U$. mediterranea, which may cause problems.

(3) U. mediterranea has rather "gentle" costae, which are widely spaced, and continuous. $U$. peregrina has very sharp costae, less distance between them. The costae are very often crenulated, because from an evolutionary point of view ( $U$. peregrina is very far from $U$. mediterranea); the costae have formed out of a series of spines. This difference can particularly well be seen on the basis of the test (first chamber); this is completely smooth in $U$. mediterranea, and uneven, covered with very small spines in $U$. peregrina.

(4) $U$. peregrina may have spinose ornementation (the last chamber, or the surface between the costae), this can almost always be detected on the first chamber (see before); $U$. mediterranea has never such spinose ornementation.

(5) Although not a $100 \%$ characteristic, the neck of $U$. mediterranea is better developed, and very often there is a prominent lip.

(6) Again not a $100 \%$ characteristic: the neck of $U$. mediterranea is very often placed in a kind of depression; the aperture of $U$. peregrina is always $100 \%$ terminal, on the highest point of the test.

Characteristic species from the outer-shelf and slope environments of the Bay of Biscay were identified by using designation and references to plates and figures in literature on Atlantic and Mediterranean foraminifera (see Table 4).

\begin{tabular}{ll}
\hline Species & References \\
\hline & \\
Amphicoryna scalaris (Batsch), 1791 & Jones (1994), pl. 63, Figs. 28-31 \\
Bigenerina nodosaria d'Orbigny, 1826 & Jones (1994), pl. 44, Figs. 19-24 \\
Biloculinella irregularis (d'Orbigny) 1839 & d'Orbigny (1839), pl. 8, Figs. 20 and 21 \\
Bolivina alata (Seguenza), 1862 & Schiebel (1992), pl. 1, Fig. 2 \\
\hline
\end{tabular}


Table 4 (continued)

\begin{tabular}{|c|c|}
\hline Species & References \\
\hline Bolivina spathulata (Williamson), 1858 & Jorissen (1987), pl. 1, Fig. 5 \\
\hline Bolivina subaenariensis Cushman, 1922 & Phleger et al. (1953), pl. 7, Figs. 24 and 25 \\
\hline Bolivinita quadrilatera (Schwager), 1866 & Jones (1994), pl. 42, Figs. 8-12 \\
\hline Bulimina costata d'Orbigny, 1826 & Van Leeuwen (1989), pl. 8, Figs. 2 and 3 \\
\hline Bulimina inflata Seguenza, 1862 & Van Leeuwen (1989), pl. 8, Fig. 4 \\
\hline Cancris auriculus (Fichtel \& Moll), 1942 & Jones (1994), pl. 106, Fig. 4 \\
\hline Cassidulina carinata Silvestri, 1896 & Phleger et al. (1953), pl. 9, Figs. 32-37 \\
\hline Chilostomella oolina Schwager, 1878 & Jones (1994), pl. 55, Figs. 12-14 \\
\hline Cibicides lobatulus Walker and Jacob, 1798 & Jones (1994), pl. 92, Fig. 10 \\
\hline Cibicides wuellerstorfi (Schwager), 1866 & Van Leeuwen (1989), pl. 10, Figs. 1-9 \\
\hline Cibicidoides pachydermus (Rzehac), 1886 & Jones (1994), pl. 94, Fig. 9 \\
\hline Cibicidoides robertsonianus (Brady), 1881 & Van Leeuwen (1989), pl. 9, Figs. 1-3 \\
\hline Clavulina cylindrica d’Orbigny, 1952 & Hofker (1932), Figs. 18 and 19 \\
\hline Cribrostomoides subglobosum (M. Sars), 1868 & Jones (1994), pl. 34, Figs. 8-10 \\
\hline Cyclammina cancellata Brady, 1879 & Jones (1994), pl. 37, Figs. 8-16 \\
\hline Cystammina pauciloculata (Brady), 1879 & Jones (1994), pl. 41, Fig. 1 \\
\hline Dentalina ariena Patterson and Pettis, 1986 & Jones (1994), pl. 62, Figs. 27-31 \\
\hline Elphidium advenum Cushman, 1922 & Phleger et al. (1953), pl. 6, Fig. 15 \\
\hline Gavelinopsis translucens (Phleger \& Parker), 1951 & Schiebel (1992), pl. 4, Fig. 5 \\
\hline Glandulina ovula d'Orbigny, 1846 & Jones (1994), pl. 61, Figs. 17-22 \\
\hline Globobulimina affinis d'Orbigny, 1826 & Phleger et al.(1953), pl. 6, Fig. 32 \\
\hline Glomospira charoides Jones \& Parker, 1860 & Phleger et al.(1953), pl. 5, Fig. 1 \\
\hline Glomospira gordialis Jones \& Parker, 1860 & Phleger et al.(1953), pl. 5, Fig. 2 \\
\hline Gyroidina altiformis Stewart \& Stewart, 1930 & Jorissen (1987), pl. 1, Fig. 11 \\
\hline Gyroidina umbonata (Silvestri), 1898 & Parker (1958), pl. 3, Figs. 19 and 20 \\
\hline Hanzawaia boueana (d'Orbigny), 1846 & Jorissen (1987), pl. 3, Fig. 10 \\
\hline Hoeglundina elegans (d'Orbigny), 1826 & Phleger et al.(1953), pl. 9, Figs. 24 and 25 \\
\hline Hyalinea balthica (Schroeter), 1783 & Jones (1994), pl. 112, Fig. 1 and 2 \\
\hline Karreriella bradyi (Cushman), 1911 & Jones (1994), pl. 41, Figs. 1-4 \\
\hline Lenticulina gibba d'Orbigny, 1839 & Jones (1994), pl. 69, Figs. 8 and 9 \\
\hline Lenticulina peregrina (Schwager), 1866 & Cushman and McCulloch (1950), pl. 39, Fig. 5 \\
\hline Melonis barleeanus (Williamson), 1858 & Van Leeuwen (1989), pl. 13, Figs. 1 and 2 \\
\hline Nonion scaphum (Fichtel and Moll), 1798 & Jones (1994), pl. 109, Fig. 12 \\
\hline Nonionella turgida (Williamson), 1858 & Jones (1994), pl. 109, Figs. 17-19 \\
\hline Nouria polymorphinoides Heron-Allen \& Earland, 1914 & Loeblich and Tappan (1988a, b), pl. 123, Figs. 11 and 12 \\
\hline Nuttallides umboniferus (Cushman), 1933 & Van Leeuwen (1989), pl. 15, Figs. 11-13; pl. 16, Figs. 1-7 \\
\hline Oridorsalis umbonatus Reuss, 1851 & Van Leeuwen (1989), pl. 17, Figs. 1-13 \\
\hline Pseuclavulina crustata Cushman, 1936 & Jorissen (1987), pl. 1, Fig. 1 \\
\hline Pseudoeponides falsobeccarii Rouvillois, 1974 & Jorissen (1987), pl. 4, Fig. 3 \\
\hline Pullenia bulloides (d’Orbigny), 1826 & Phleger et al.(1953), pl. 10, Fig. 19 \\
\hline Pyrgo depressa (d’Orbigny), 1826 & Jones (1994), Pl. 2, Figs. 12, 16 and 17 \\
\hline Pyrgo subsphaerica d’Orbigny, 1839 & Cushman (1929), pl. 18, Figs. 1and 2 \\
\hline Pyrgoella sphaera (d'Orbigny), 1839 & Jones (1994), pl. 2, Fig. 4 \\
\hline Quinqueloculina seminula (Linné), 1758 & Jones (1994), pl. 5, Fig. 6 \\
\hline Rectuvigerina phlegeri Le Calvez, 1959 & Le Calvez (1959), pl. 1, Fig. 11 \\
\hline Reophax ampullacea Brady, 1881 & Jones (1994), pl. 30, Fig. 6 \\
\hline Reophax scorpiurus Montfort, 1808 & Loeblich and Tappan (1988a, b), pl. 44, Figs. 1-3 \\
\hline Sigmoilopsis schlumbergeri Silvestri, 1904 & Jones (1994), pl. 8, Figs. 1-4 \\
\hline Siphotextularia affinis Fornasini, 1883 & Kohl (1985), pl. 2, Fig. 5 \\
\hline Textularia agglutinans d'Orbigny, 1839 & Jones (1994), pl. 43, Figs 1-3 \\
\hline Textularia sagittula Defrance, 1824 & Jorissen (1987), pl. 3, Fig. 12 \\
\hline Trifarina angulosa (Williamson), 1858 & Jones (1994), pl. 74, Figs. 17 and 18 \\
\hline Trifarina pauperata (Heron-Allen \& Earland), 1932 & Timm (1992), pl. 6, Fig. 4 \\
\hline
\end{tabular}


Table 4 (continued)

\begin{tabular}{ll}
\hline Species & References \\
\hline Trochammina inflata (Montagu), 1808 & Jones (1994), pl. 41, Fig. 4 \\
Uvigerina elongatastriata (Colom), 1952 & Van der Zwaan et al. (1986), pl. 6, Figs. 1-8 \\
Uvigerina mediterranea Hofker, 1932 & Van der Zwaan et al. (1986), pl. 5, Figs. 1-7 \\
Uvigerina peregrina Cushman, 1923 & Van der Zwaan et al. (1986), pl. 1, Figs. 1-6 \\
Uvigerina proboscidea Schawger, 1866 & Van der Zwaan et al. (1986), pl. 12, Figs. 1-4 \\
Valvulineria bradyana (Fornasini), 1900 & Jorissen (1987), pl. 4, Figs 1 and 2 \\
\hline
\end{tabular}

\section{References}

Altenbach, A.V., 1988. Deep sea benthic foraminifera and flux rate of organic carbon. Revue de Paléobiologie 2, 719-720.

Altenbach, A.V., Sarnthein, M., 1989. Productivity record in benthic foraminifera. In: Berger, W.H., Smetacek, V.S., Wefer, G. (Eds.), Productivity of the Ocean: Present and Past. Wiley, Chichester, pp. 255-269.

Alve, E., Bernhard, J.M., 1995. Vertical migratory response of benthic foraminifera to controlled oxygen concentrations in an experimental mesocosm. Marine Ecology Progress Series $116,137-151$.

Anderson, L., 1979. Simultaneous spectrophotometric determination of nitrite and nitrate by flow injection analysis. Analytica Chemica Acta 110, 123-128.

Anschutz, P., Hyacinthe, C., Carbonel, P., Jouanneau, J.M., Jorissen, F.J., 1999. La distribution du phosphore inorganique dans les sédiments modernes du Golfe de Gascogne. Comptes Rendus Academie Sciences, Paris 328, 765-771.

Barmawidjaja, D.M., Jorissen, F.J., Puskaric, S., Van der Zwaan, G.J., 1992. Microhabitat selection by benthic foraminifera in the northern Adriatic Sea. Journal of Foraminiferal Research 22 (4), 297-317.

Barnett, P.R.O., Watson, J., Connely, D., 1984. A multiple corer for taking virtually undisturbed sample from shelf, bathyal and abyssal sediments. Oceanologica Acta 7, 399408.

Berger, W.H., Diester Haas, L., 1988. Paleoproductivity; the benthic/planktonic ratio in foraminifera as a productivity index. Marine Geology 81, 1-4.

Berger, W.H., Wefer, G., 1990. Export productivity: seasonality and intermittency, and paleooceanographic implications. Palaeogeography, Palaeoclimatology, Palaeoecology 9, 245-254.

Bernhard, J.M., 1988. Postmortem vital staining in benthic foraminifera: duration and importance in population and distributional studies. Journal of Foraminiferal Research 18, 143-146.

Bernhard, J.M., Alve, E., 1996. Survival, ATP pool, and ultrastructural characterization of benthic foraminifera from Drammensfjord (Norway); response to anoxia. Marine Micropaleontology 28 (1), 5-17.

Bernhard, J.M., Sen Gupta, B., 1999. Foraminifera of oxygendepleted environments. In: Sen Gupta, B.K. (Ed.), Modern Foraminifera. Kluwer Academic Press, Dordrecht.
Buzas, M., Culver, S.J., Jorissen, F.J., 1993. A statistical evaluation of the microhabitats of living (stained) infaunal benthic foraminifera. Marine Micropaleontology $20,3-4$.

Carney, R.S., 1989. Examining relationships between organic carbon flux and deep-sea deposit feeding. In: Lopez, G., Taghon, G., Levinton, J. (Eds.), Ecology of Marine Deposit Feeders, Vol. 31. Springer, Lecture Notes on Coastal and Estuarine Studies, pp. 24-58.

Corliss, B.H., 1985. Microhabitats of benthic foraminifera within deep-sea sediments. Nature 314, 435-438.

Corliss, B.H., 1991. Morphology and microhabitat preferences of benthic foraminifera from the northwest Atlantic Ocean. Marine Micropaleontology 17, 195-236.

Corliss, B.H., Chen, C., 1988. Morphotype patterns of Norwegian Sea deep-sea benthic foraminifera and ecological implications. Geology 16, 716-719.

Corliss, B.H., Emerson, S., 1990. Distribution of Rose Bengal stained deep-sea benthic foraminifera from the Nova Scotia continental margin and Gulf of Maine. Deep-Sea Research 37, 381-400.

Coull, B.C, Ellison, R.L., Fleeger, J.W., Higgins, R.P., Hope, W.D., Hummon, W.D., Rieger, R.M., Sterrer, W.E., Thiel, H., Tietjen, J.H., 1977. Quantitative estimates of the meiofauna from the deep sea off North Carolina, USA. Marine Biology 39, 233-240.

Cushman, J.A., 1929. The Foraminifera of the Atlantic Ocean. Part 6. Miliolidae, Ophtalmidiidae and Fischerinidae. Smithsonian Institution. United States National Museum. Bulletin 104.

Cushman, J.A., McCulloch, I., 1950. Some virgulininae in the collections of the Allan Hancock foundation. Alan Hancock Pacific Expedition 6, 295-364.

De Rijk, S., Jorissen, F.J., Rohling, E.J., Troelstra, S.R., 2000. Organic flux control on bathymetric zonation of Mediterranean benthic foraminifera. Marine Micropaleontology 40, 151-166.

Fariduddin, M., Loubere, P., 1997. The surface ocean productivity response of deeper water benthic foraminifera in the Atlantic Ocean. Marine Micropaleontology 32, 289-310.

Fenchel, T.M., Finlay, B.J., 1995. Ecology and Evolution in Anoxic Worlds. Oxford University Press, Oxford, 276pp.

Fenchel, T.M., Jørgensen, B.B., 1977. Detritus food chains of aquatic ecosystems: the role of bacteria. Advances in Microbial Ecology 1, 1-58. 
Fernandez, E., 1990. Composicion, distribucion y produccion del fitoplancton en el Cantabrico Central. Thesis doctoral, Universitad de Oviedo, 388pp.

Fernandez, E., Boyd, P., Holligan, P.M., Harbour, D.S., 1993. Production of organic and inorganic carbon within a largescale coccolithophore bloom in the northeast Atlantic Ocean. Marine Ecology Program Series 9, 271-285.

Fernandez, E., Maranon, E., Cabal, J., Alvarez, F., Anadon, R., 1995. Vertical particle flux in outer shelf waters of the southern Bay of Biscay in summer 1993. Oceanologica acta 18 (3), 379-384.

Fontanier, C., Jorissen, F.J., David, C., Anschutz, P., Chaillou, G., Lafon, V. Seasonal and inter-annual variability of benthic foraminiferal fauna at $550 \mathrm{~m}$ depth in the Bay of Biscay, in preparation.

Froelich, P.N., Klinkhammer, G.P., Bender, M.L., Luedke, N.A., Heath, G.R., Cullen, D., Dauphin, P., Hammond, D., Hartman, B., Maynard, V. 1979. Early oxidation of organic matter in pelagic sediments of the Eastern Equatorial Atlantic: suboxic diagenesis. Geochemica et Cosmochimica Acta 43, 1075-1090.

Gooday, A., 1993. Deep-sea benthic foraminifera species which exploit phytodetritus: characteristic features and controls on distribution. Marine Micropaleontology 22, 187-205.

Gooday, A., Levin, L.A., Linke, P., Heeger, T., 1992. The role of benthic foraminifera in deep-sea food webs and carbon cycling. In: Rowe, G.T., Patiente, V. (Eds.), Deep-sea Food Chains and the Global Carbon Cycle. Kluwer Academic publishers, The Netherlands, pp. 63-91.

Hargrave, B.T., 1970. The utilization of benthic microflora by Hyalella azteca (Amphipoda). Journal of Animal Ecology 39, 427-437.

Harloff, J., Mackensen, A., 1997. Recent benthic foraminiferal associations and ecology of the Scotia Sea and Argentine Basin. Marine Micropaleontology 31, 1-29.

Helder, W., Bakker, J.F., 1985. Shipboard comparison of micro- and mini electrodes for measuring oxygen in marine sediments. Limnology and Oceanography 30, 11061109.

Herguera, J.C., 1992. Deep-sea foraminifera and biogenic opal: glacial to postglacial productivity changes in the western Pacific. Marine Micropaleontology 19, 79-98.

Herguera, J.C., Berger, W.H., 1991. Paleoproductivity from benthic foraminifera abundance; glacial and postglacial change in the west-equatorial Pacific. Abstracts with Program Geological Society of America 23 (5), 107.

Hermelin, J.O.R., Shimmield, B.G., 1990. The importance of the oxygen minimum zone and sediment geochemistry in the distribution of recent benthic foraminifera in the Northwest Indian Ocean. Marine Geology 91, 1-1029.

Heussner, S., Durrieu de Madron, X., Radakovitch, O., Beaufort, L., Biscaye, P.E., Carbonne, J., Delsaut, N., Etcheber, H., Monaco, A., 1999. Spatial and temporal patterns of downward particle fluxes on the continental slope of the Bay of Biscay (northeastern Atlantic). Deep-sea Research II 46, 2101-2146.
Hofker, J., 1932. Notizen ueber die Foraminiferen des Golfes von Neapel. III. Die foraminiferen fauna der Ammontatura. Pubblicazioni della Stazione Zoologica di Napoli, Vol. 12, Fasc. 1.

Hyacinthe, C., Anschutz, P., Carbonel, P., Jouanneau, J.M., Jorissen, F.J., 2001. Early diagenetic processes in the muddy sediments of the Bay of Biscay. Marine Geology 177, 111-128.

Jannink, N.T., Zachariasse, W.J., Van der Zwaan, G.J., 1998. Living (Rose Bengal stained) benthic foraminifera from the Pakistan continental margin (northern Arabian Sea). DeepSea Research I 45, 1483-1513.

Jones, R.W., 1994. The Challenger Foraminifera. Oxford Science Publications-The Natural History Museum, $149 \mathrm{pp}$.

Jones, R.W., Charnock, M.A., 1985. "Morphogroups" of agglutinating foraminifera. Their life positions and feeding habits and potential applicability in (paleo)ecological studies. Revue de Paléobiologie 4, 311-320.

Jorissen, F.J., 1987. The distribution of benthic foraminifera in the Adriatic Sea. Marine Micropaleontology 12, 21-48.

Jorissen, F.J., 1988. The distribution of benthic foraminifera from the Adriatic Sea; principles of phenotypic variation. Utrecht Micropaleontological Bulletin 37, 176.

Jorissen, F.J., 1999. Benthic foraminiferal microhabitats. In: Sen Gupta, B.K. (Ed.), Foraminifera. Kluwer Academic Publishers, Dordrecht, pp. 161-179.

Jorissen, F.J., de Stigter, H.C., Widmark, J.G.V., 1995. A conceptual model explaining benthic foraminiferal microhabitats. Marine Micropaleontology 22, 3-1715.

Jorissen, F.J., Wittling, I., Peypouquet, J.P., Rabouille, C., Relexans, J.C., 1998. Live benthic foraminiferal faunas off Cap Blanc, NW Africa: community structure and microhabitats. Deep-Sea Research I 45, 2157-2188.

Jorissen, F.J., Abu-Zaid, R.H., Bonnin, J., Crihan, M., Fontanier, C., Rohling, E.J. Benthic foraminiferal extinction patterns in Mediterranean sapropels, in preparation.

Kitazato, H., 1994. Foraminiferal microhabitats in four marine environment around Japan. Marine Micropaleontology 24, $29-41$.

Kohl, B., 1985. Early pliocene benthic foraminifers from the Salina Basin, Southeastern Mexico. Bulletins of American Paleontology 88/322, 1-173.

Laborde, P., Urrutia, J., Valencia, V., 1999. Seasonal variability of primary production in the Cap-Ferret Canyon area (Bay of Biscay) during the ECOFER cruises. Deep-Sea Research II 46, 2057-2079.

Le Calvez, 1959. Recents Travaux de l'Institut des Peches Maritimes Vol. 23, 263pp.

Le Corre, P., Treguer, P., 1976. Caractéristiques chimiques et planctoniques du Golfe de Gascogne et du Proche Atlantique. Campagne POLYGAS A $(20.10$ au 04.11.1972) et PHYGAS 32 (24.04 au 08.05.1973). Résultats des campagnes à la mer CNEXO 9, 306.

Lee, J.J., 1979. Nutrition and physiology of the foraminifera. In: Levandowsky, M., Hutner, S.H. (Eds.), Biochemistry and Physiology of Foraminifera. Academic Press, New York, pp. 42-66. 
Le Floch, J., 1968. Sur la circulation de l'eau d'origine méditerranéenne dans le golfe de gascogne et ses variations à courte période. Cahiers Océanographiques XX(7), 653-661.

Linke, P., Lutze, G.F., 1993. Microhabitats preferences of benthic foraminifera: a static concept or a dynamic adaptation to optimize food acquisition? Marine Micropaleontology 20, 215-234.

Loeblich, A.R., Tappan, H., 1988a. Foraminifera Genera and their Classification. Van Nostrand Reinhold, New York, 970pp.

Loeblich, A.R., Tappan, H., 1988b. Foraminifera Genera and their Classification-Plates. Van Nostrand Reinhold, New York, 212pp.

Loubere, P., Anthony, G., Lagoe, M., 1993. Sea-bed biogeochemistry and benthic foraminiferal bathymetric zonation on the slope of the northwest Gulf of Mexico. Palaios 8, 439-449.

Lutze, G., Coulbourn, W., 1984. Recent benthic foraminifera from the continental margin off northwest Africa: community structure and distribution. Marine Micropaleontology 8, 361-401.

Moodley, L., Van der Zwaan, G.J., Herman, P.M., Kempers, A.J., van Breugel, P., 1997. Differential response of benthic meiofauna to anoxia with special reference to Foraminifera (Protista: Sarcodina). Marine Ecology Progress series 158, 151-163.

Morigi, C., Jorissen, F.J., Gervais, A., Guichard, S., Borsetti, A.M. Benthic foraminiferal faunas in surface sediments off NW Africa: relationship with the organic flux to the ocean floor. Journal of Foraminiferal Research, in press.

Mullins, H.T., Thompson, J.B., McDougall, K., LVercoutere, T., 1985. Oxygen minimum zone edge effects: evidence from the central California coastal upwelling system. Geology 13, 491-494.

Ogawa, N., Tauzin, P., 1973. Contribution à l'étude hydrologique et géochimique du golfe de cap-breton. Bulletin de l'institut Géologique du Bassin d'Aquitaine, Bordeaux 14, $19-46$.

Ohga, T., Kitazato, H., 1997. Seasonal changes in bathyal foraminiferal populations in response to the flux of organic matter (Sagami Bay, Japan). Terra Nova 9, 33-37.

d'Orbigny, A., 1839. Voyage dans l'amérique méridionale. Foraminifères $5,86$.

Parker, F.L., 1958. Eastern Mediterranean Foraminifera. Reports of the Swedish deep-sea Expedition 1947-1948, Vol. VIII: Sediment Cores from the Mediterranean and the Red Sea, No. 4, 283pp.

Phleger, F.B., Parker, F.L., Peirson, J.F., 1953. North Atlantic Foraminifera. Reports of the Swedish Deep-sea Expedition,VOL. VII: Sediment Cores from the North Atlantic, No. 1, 122pp.

Pingree, R.D., Mardell, G.T., New, A.L., 1986. Propagation of internal tides from the upper slopes of the Bay of Biscay. Nature 321, 154-158.

Rathburn, A.E., Corliss, B.H., 1994. The ecology of living (stained) deep-sea benthic foraminifera from the Sulu Sea. Paleoceanography 9, 87-150.
Revsbech, N.P., 1983. In-situ measurements of oxygen profiles of sediments by use of oxygen microelectrodes. In: Ganuger Forstner, Polarographic Oxygen Sensors. Springer, Berlin, pp. 265-273.

Revsbech, N.P., Jørgensen, B.B., 1986. Microelectrodes: their use in microbial ecology. Advances in Microbial Ecology 9, 243-252.

Schiebel, R., 1992. Rezente benthische Foraminiferen in Sedimenten des Schelfes und oberen Kontinentalhanges im Golf von Guinea (Westafrika). Berichte-Reports Geologisch-Paläontologisches Institut und Museum ChristianAlbrechts-Universität Kiel 51, 126.

Sen Gupta, B.K., Aharon, P., 1994. Benthic foraminifera of bathyal hydrocarbon vents of the Gulf of Mexico: initial report on communities and stable isotopes. Geo-marine Letters 14, 88-96.

Sen Gupta, B.K., Machain-Castillo, M.L., 1993. Benthic foraminifera in oxygen-poor habitats. Marine Micropaleontology 20, 3-4.

Stookey, L.L., 1970. Ferrozine-a new spectrophotometric reagent for iron. Analytical Chemistry 42, 779-781.

Strickland, J.D.H., Parsons, T.R., 1972. A practical handbook of seawater analysis. Bulletin of Fisheries Resource B Canada 167, 311.

Thiel, H., 1983. Meiobenthos and nanobenthos of the deep sea. In: Rowe, G. (Ed.), Deep Sea Biology, The sea, Vol. 8. Wiley Interscience, New York, pp. 167-230.

Thomsen, L., Altenbach, A.V., 1993. Vertical and areal distribution of foraminiferal abundance and biomass in microhabitats around inhabited tubes of marine echiurids. Marine Micropaleontology 20, 303-309.

Tietjen, J.H., 1971. Ecology and distribution of deep-sea meiobenthos off North Carolina. Deep-Sea Research 18, 941-957.

Timm, S., 1992. Rezente Tiefsee-Benthosforaminiferen aus Oberflächen-sedimenten des Golfes von Guinea (Westafrika) - Taxonomie, Verbreitung, Ökologie und Korngrößenfraktionen. Berichte-Reports, Geologisch-Paläontologisches Institut und Museum Christian-Albrechts-Universität Kiel 59, 155.

Tréguer, P., Le Corre, P., Grall, J.R., 1979. The seasonal variations of nutrients in the upper waters of the Bay of Biscay region and their relation to phytoplanctonic growth. Deep-Sea Research 26 (10A), 1121-1152.

Van der Zwaan, G.J., 1982. Paleoecology of Late Miocene Mediterranean Foraminifera. Utrecht Micropaleontological Bulletin 25, 201.

Van der Zwaan, G.J., Jorissen, F.J., 1991. Biofacial patterns in river-induced shelf anoxia. In: Tyson, R.V., Pearson, T.H. (Eds.), Modern and Ancient Continental Shelf Anoxia, Vol. 58. Geological Society Special Publication, pp. 65-82.

Van der Zwaan, G.J., Jorissen, F.J., Verhallen, P.J.J.M., Von Daniels, C.H., 1986. Atlantic-European Oligocene to recent Uvigerina: taxonomy, paleoecology and paleobiogeography. Utrecht Micropaleontological Bulletins 35, 240.

Van der Zwaan, G.J., Duijnstee, I.A.P., den Dulk, M., Ernst, S.R., Jannink, N.T., Kouwenhoven, T.J., 1999. Benthic foraminifers: proxies or problems? A review of paleoecologial concepts. Earth-Sciences Reviews 46, 213-236. 
Van Leeuwen, R.J.W., 1989. Sea-floor distribution and Late Quaternary faunal patterns of planktonic and benthic foraminifers in the Angola Basin. Utrecht Micropaleontological Bulletins 38, 288.

Verhallen, P.J.J.M., 1987. Early development of Bulimina marginata in relation to paleoenvironmental changes in the Mediterranean. Proceedings Koninklijke Nederlandse Akademie van Wetenschappen, Series B 90, 161-180.

Walton, W.R., 1952. Techniques for recognition of living Foraminifera. Contribution from the Cushman Foundation the Foraminiferal Research 3, 56-60. 